\title{
Coordination through Reputations: A Laboratory Experiment*
}

\author{
Donald J. Dale John Morgan $^{\ddagger} \quad$ Robert W. Rosenthal ${ }^{\S}$ \\ Revised March 2000
}

\begin{abstract}
One of the functions of reputations in societies is to enable coordination, and some of the most familiar instances of this occur when reputations are based on past actions. For example, a history of aggressive play is often interpreted as predicting more aggressive play in the future. This permits a pair of individuals with unequal reputations for aggressiveness to avoid wasteful conflict that would result from aggressive play by both.

This paper describes a laboratory experiment designed to explore whether, in a population of subjects, such coordination through reputation formation will emerge spontaneously, what form it will take, and how reliably it will be observed by the individuals in the population. Reputation-based coordination of the sort illustrated above did arise in all experimental sessions, but the rate of adherence to the social rule implicitly adopted by the group varied across the experimental populations as a function of treatment variables.
\end{abstract}

${ }^{*}$ We are grateful to a referee, to participants at seminars at M.I.T. and Boston University for helpful suggestions, to the National Science Foundation for financial support, and to Sumon Majumdar for research assistance. Data from these experiments is downloadable (in Stata 6.0 format) at www.wws.princeton.edu/ rjmorgan.

$\dagger$ Department of Economics, Princeton University

$\ddagger$ Department of Economics and the Woodrow Wilson School, Princeton University

$\S$ Department of Economics, Boston University 


\section{Introduction}

The idea that an individual might wish to condition her actions on the reputations of the people with whom she is interacting is ubiquitous in human societies, and the functions such reputational systems can serve are numerous. In this paper, we study one of the familiar functions of reputationsthat of a coordination device. The basic idea is that if two individuals must make simultaneous decisions in a symmetric situation and if there is some advantage to at least one of the individuals if they act differently from each other, then any external cues, if agreed upon either tacitly or explicitly, can serve to direct them toward a coordinated asymmetric outcome. Reputations can provide such external cues. As an analogy, many species of animals are observed to respect "pecking orders" which peacefully desymmetrize situations that might otherwise result in conflict. (A typical example is males competing for the opportunity to copulate with all the fertile females in a herd. The top male often wins without a fight). For a human example, an individual (or firm) having the reputation of a bully may be able to avoid conflict and prosper because potential competitors either defer to the bully when in situations of conflict or avoid such situations to begin with.

There are complications even in these relatively simple examples, however. Is the top animal in the pecking order the individual with the toughest appearance (e.g., largest size, sharpest antlers, brightest color) or the one with the best record in past conflicts? How many instances of bullylike behavior does it take for the reputation of bully to be acquired and how long does it last? In short, what is the nature of the external cue? In real-world situations the natures of external cues can differ for reasons having much more to do with example-specific details than with the coordinating role of the cue. For example, the ability to remember the identity of the strongest or weakest animal in one's herd might have survival value in the face of threats from external predators or might be useful in finding food. Similarly, bully reputations might correlate with aggressive tendencies toward external foes; and it might therefore pay either to align with or against bullies in one's own society.

To learn about how humans use reputations as coordinating devices, therefore, it seems useful to explore the question in the laboratory, where many of these other factors can be controlled for, at least to some extent. In this paper we report on one such experiment. Our hope is that the aspects of everyday behavior that concern the coordinating role of reputations are 
maintained in experimental behavior despite the abstract setting of the laboratory environment. We know of no previous experiments on this subject.

We report here on the experimental results from a variety of sequenced $2 \times 2$ coordination games with successively rematched player pairs, where prior to play in each round each pair of subjects was presented with a pair of summary signals, called "current positions," that reveal information about the recent play of both members of the pair. We did not describe the positions to the subjects as reputations and did not suggest to the subjects that conditioning on positions was appropriate. We merely gave the group of subjects the opportunity to develop, collectively but implicitly (since they were given no opportunities to communicate directly with each other), a coordination mechanism by providing to each matched pair of players the common knowledge of their respective current positions. ${ }^{1}$ Our interest was in whether, how, and how reliably, our experimental societies would use the position pairs as coordinating devices and in which of the many possible position-based coordinating rules they would adopt.

More specifically, the subjects in a session played repeatedly the same variant of either

\begin{tabular}{|c|c|}
\hline 0,0 & $x-y, x+y$ \\
\hline$x+y, x-y$ & 0,0 \\
\hline
\end{tabular} or \begin{tabular}{|c|c|}
\hline$x, x$ & $x-y, x+y$ \\
\hline$x+y, x-y$ & 0,0 \\
\hline
\end{tabular}

where $x>y \geq 0$. Identifying the top row with the left column, all such oneshot games are seen to be symmetric, but two of their Nash equilibria - the ones that use pure strategies - are not symmetric. Furthermore, though the third Nash equilibrium in each such game is symmetric, it involves an inefficient mixed-strategy combination. Each subject's position is a signal about how frequently in the recent past she has played the bottom row (equivalently, the right column) which we might think of as the more aggressive action.

Our prior guess was that the incentives to coordinate on the pure Nash equilibria of the one-shot games would be sufficiently strong that the subjects would find ways to achieve them quite frequently. Furthermore, we suspected that the organizing rule with the most salience would be that in cases where a matched pair finds itself in unequal positions the player having the "tougher" current position (i.e., the position signalling more frequent recent play of

\footnotetext{
${ }^{1}$ Of course there might be an experimenter effect: subjects might try to use their positions to coordinate solely because they are prominently available in the experiment.
} 
the aggressive action) would play the aggressive strategy while the player with the "softer" current position would choose the other action. This seems to accord with everyday intuition: individuals who have reputations formed from their records of previous actions are commonly expected to continue as in the past. But why this rule is so intuitive is itself not obvious; after all, the reverse rule (tougher reputation types playing soft and softer types playing tough) coordinates similarly. In addition, there are many other patterns which, though more complicated in that the identity of the player chosen to play tough depends on more details of the state pairs, would also achieve coordination.

From a theoretical standpoint, there is little basis for distinguishing among various organizing rules. For instance, Rosenthal and Landau (1979) examine whether the intuitive rule might have an evolutionary advantage (measured by population coordination rates) over the reverse rule. They find, however, that no systematic evolutionary advantage accrues to a population equilibrium involving the intuitive rule as compared to one involving the reverse rule. And the broader question of what the optimal equilibrium rule is from an efficiency perspective remains open. In view of the lack of guidance for population rule selection in the theoretical literature, an important contribution of our study is to assess experimentally to what extent experimental subject populations behave as though they have coordinated on a rule and, if yes, which rule.

Absent either an evolutionary or an efficiency rationale, one can speculate about other reasons that might lead to the adoption of the intuitive rule. Perhaps it is the simplicity of the intuitive rule that makes it salient (although the reverse rule seems equally simple). Perhaps it is the fact that people who play according to the simplest of all rules - constantly the same pure strategy - end up in the extreme positions and therefore are not observed to violate the intuitive rule. And perhaps our prior guesses would be proved wrong what seems focal to us is not necessarily focal to everyone, and if there is heterogeneity in intuition across the population, this may make adoption of any one rule difficult for the subject population.

Our experimental findings have turned out to be quite interesting. We ran sixteen sessions, all with initially inexperienced subjects, for 100 rounds each. Ten of our sessions were based on parametric variants of "Battle of the Sexes," or BOS, the first game form above; and six used parametric variants of "Hawk-Dove" or "Chicken," or HD, the second game form above. Examining the last 50 rounds of each of the sessions, we find that, to the extent that 
any one simple rule explains play, it is overwhelmingly the intuitive rule. In the BOS sessions, when a player is matched with a co-player in an unequal position, she plays according to the intuitive rule $90.6 \%$ of the time. The intuitive rule is somewhat less successful in the HD sessions, but it still predicts $78.9 \%$ of choices correctly for players matched with an unequally positioned opponent. Both satisficing and altruistic motives could be explanations for this difference across game forms: The less aggressive strategy is relatively safe in HD, and if its payoff against either strategy of the opponent is good enough, why risk it for a small gain? This safe strategy is also kindly toward the opponent no matter what he does.

The compliance percentages from unequal positions listed above are averages over treatments for each game form in which the $y$-payoff parameter was varied. In both BOS and HD treatments, increasing the $y$-payoff parameter unambiguously reduced the predictive power of the intuitive rule. When $y=0$ in BOS, the game becomes a pure coordination game and the intuitive rule correctly predicted $97.8 \%$ of choices; this declined to $90.8 \%$ when a small positive $y$ was used and still farther to $81.4 \%$ in the treatment having the highest $y$ value. The situation was similar for HD: With the same small $y$ value as in BOS, the intuitive rule correctly predicted $91.0 \%$ of choices. With the larger $y$ value, the intuitive rule predicted $72.9 \%$ of HD choices. $^{2}$

In fact, the majority of departures from the intuitive rule, especially in the BOS sessions, were by players who deviated to the aggressive strategy. This suggests a calculation involving a sacrifice of current payoff for improved future payoffs. In order to quantify such trade-offs and thereby attempt to explain the varying predictive power of the intuitive rule across experimental sessions, however, it is useful to view the experiment through the lens of a well-specified mathematical model.

The model of Rosenthal and Landau (1979) is a relatively simple lens through which to view this experiment. It depicts a society consisting of a large number of individuals who are repeatedly randomly matched to play a game (having von-Neumann-Morgenstern utility payoffs) over an infinite time sequence, where all individuals are assumed to discount according to a common fixed factor. ${ }^{3}$ Actions produce effects on payoff-irrelevant individual state variables, and matched pairs of individuals know their own and

\footnotetext{
${ }^{2} \mathrm{HD}$ with $y=0$ is a game with different equilibrium structure, so we did not try any such treatments.

${ }^{3}$ Rosenthal and Landau restricted their analysis to a particular parametrization of HD, but their model is usable more generally.
} 
opponents' individual states, just as in our experiment the matched pairs of subjects know both positions. A steady state equilibrium of this model is a stationary Markov rule that assigns to each ordered state pair ((own, opponent's)) a strategy (possibly mixed) in the stage game such that each used action is a best response in each state pair, taking the future into account as follows: The states occupied by an individual's future matched opponents will be i.i.d. draws from the unique invariant distribution over individual states generated by the Markov rule, and all individuals will play according to the rule in the future. Furthermore, an extension of the intuitive rule (so that it also makes choices in equal-position situations) generates one of the steady state equilibria of the model when the common discount factor is not too high relative to the parameter $y$; and the smaller is $y$, the larger is the set of common discount factors for which the intuitive rule generates a steady state equilibrium.

The applicability of this model to the experiment is evidently suspect because of its simplifying assumptions of an infinite future, discounting, money payoffs being von-Neumann-Morgenstern utilities, and an infinite player set. Despite these, the equilibrium of the model corresponding to the intuitive rule seems to organize the data reasonably well. (We shall discuss later why the assumptions above might not seriously misrepresent our subjects' behavior.) So, although the relationship between the model and the experiment seems crude, it fits the data reasonably well in many respects, provides at least a hint of an explanation for the observed effect of the $y$ parameter, and therefore leads us to the following tentative conclusions.

1. Of the model's many steady state equilibria, only the one corresponding to the intuitive rule approximates play observed in the laboratory.

2. Experimental play of the intuitive rule is most reliable in BOS with small $y$ parameter and least reliable in HD with high $y$ parameter; and, within each game form, reliability is monotone in $y$.

3. Since for any fixed common discount factor and fixed game form the intuitive rule generates a steady state equilibrium only when $y$ is sufficiently small, the relatively high noise levels in the data for the experimental sessions with larger $y$ values reflect the increased incentives that relatively patient subjects have to deviate from the prescriptions of the intuitive rule. ${ }^{4}$

\footnotetext{
${ }^{4}$ We will discuss later why we think degrees of impatience might matter in our experi-
} 
We hasten to add that our claim that the model fits the experimental data reasonably well is inherently subjective. The intuitive rule, as formulated here, allows for no variance in play when players in unequal positions are matched. Since there is some variance in the experimental data, statistical tests would reject the hypothesis that the model could have generated the data. Furthermore, we have run only 16 sessions; and these encompass a variety of treatments. In addition, the model predicts subject play least well in situations where a subject and her matched opponent are in equal positions; i.e., in the situations where the intuition for the intuitive rule seems murkiest. Thus our findings could be interpreted more as supportive of the intuitive rule than of the intuitive rule together with the model in which we have chosen to embed it.

Because many features of the experiment and many of the questions that can potentially be addressed using its data are complex, we intend to present our findings in stages. This paper, the first such stage, is devoted to the "long run," or what happens after play settles down. This seems appropriate given our focus on first understanding the long-run phenomenon of how humans have come to use reputations as coordinating devices. We believe that our subjects have learned sufficiently about their environment by round 50 and that their behavior after that is in the aggregate approximately stationary. (We will present evidence for this as well as for the absence of end-game effects in the data.) Accordingly, in this paper we will restrict our attention almost entirely to the data from rounds 51-100 of each session. We will then compare the experimental results for these rounds with certain steady state equilibria of the theoretical model, leaving for future analysis such interesting questions as why the intuitive rule is apparently focal and how the populations of subjects learn to play it.

The rest of this paper is organized as follows. Section 2 reviews some relevant literature on the role of reputation and other signals as coordinating devices and highlights the connection of this paper with that literature. Section 3 describes the experimental design and procedures. Section 4 presents the theoretical model to be adapted to the experiment. Section 5 presents the results of our experiment and compares them to the theoretical predictions. Section 6 concludes.

ment even though all payments are made at the end of the experimental session. 


\section{Review of Literature}

Although the model of Rosenthal and Landau (1979) and the experiments here share a distinct approach to the issue, our work relates more broadly to theoretical and experimental attempts to understand how desymmetrizing opportunities may be used to enhance coordination. It is now well understood theoretically that the introduction of pre-play communication consisting of costless signals can affect the equilibrium outcomes of games (see Kreps and Sobel (1994) for a survey). Since players can condition their choices on the messages exchanged during pre-play communication, one can achieve equilibrium outcomes in the extended game that are not possible in the original game where pre-play communication is absent. For two-person, normal-form games, such as those we study in this paper, the set of Nash equilibrium payoffs in the extended game is simply the convex hull of Nash equilibrium payoffs in the original game (see Aumann and Hart (1999)). For normal-form games of four or more players, Barany (1992) has shown that the set of Nash equilibria in the extended game corresponds to the set of correlated equilibria in the original game.

Costly signaling can work as well to achieve coordination. Perhaps the most dramatic example of this is Ben-Porath and Dekel (1992), where one player's ability to burn money in advance of a BOS allows him to achieve his preferred equilibrium (under suitable forward induction logic). Interestingly, in this situation, the signal that both players condition upon to achieve this outcome is the decision not to burn money, so it is the mere availability of the costly signal, and not the incursion of the cost, that does the job.

In contrast, we study situations where the pre-play "messages," in the form of a player's current position, depend upon a player's past actions. Although our signals are costless in the sense that payoffs for a given round of the game depend only on choices and not on pre-play messages, signals are costly in the broader sense that a player's past payoff-relevant choices determine his current position in a given round of the game. Thus, the costs, such as they are, of signalling are determined endogenously in the class of games we study.

Our paper is also somewhat related to the extensive literature on focal points (see Schelling (1960) for the classic treatment). This literature seeks to understand inherent properties of a situation that may be used to achieve coordination. The current positions in our model may be viewed as such an inherent property. However, the fact that a player can consciously "build 
up" his reputation with an eye toward generating beneficial asymmetries in the future distinguishes our approach from this literature.

On the experimental front, symmetric markets have provided perhaps the best settings in which desymmetrized coordination has been examined. Ochs (1990) and Meyer, et al. (1992) study decentralized market games in which subjects simultaneously select locations at which trade can take place. And a series of experiments (e.g., Rapoport (1995) and Rapoport, et al. (1999)) studies simultaneous market entry decisions by subjects in identical circumstances where the payoffs to entry decrease in the number of subjects choosing to enter. In addition, Cooper, et al. (1994), provide BOS trials in which a prepended desymmetrizing event selects one of the equilibria with great regularity.

\section{Experimental Design and Procedures}

The experiment consisted of 16 sessions conducted at Princeton University in Spring 1999. Twelve volunteer subjects (Princeton undergraduates and/or Masters students from the Woodrow Wilson School) participated in each session, with no subjects taking part in more than one session. Subjects were recruited by e-mail invitations to participate in a decision making experiment. They were promised between $\$ 5$ and $\$ 35$ for a session lasting approximately 60 minutes. In fact, no session lasted longer than 60 minutes; the lowest payoff actually earned in the experiment was $\$ 12.70$; and the highest amount earned was $\$ 27.25$. Even the lowest amount is considerably more than the hourly outside earning rate of the typical student. The subjects were paid their winnings individually in cash at the end of the session.

The following procedures were common to all sessions. At the beginning of a session the subjects were seated at computer terminals in a single room and given a set of instructions. These instructions were read aloud, and the subjects were then given an opportunity to ask questions. (See the appendix for a sample copy of the instructions used.) Subjects then played the same symmetric $2 \times 2$ normal-form game 100 times in succession. No communication between subjects was permitted, and once play began all choices and information were transmitted via the computer terminals. In each round, each subject was randomly and anonymously paired with another individual in the session, with equal probability given to all eleven others in each round. Subjects chose between the two possible actions, one labeled " $\rightarrow$ " and the 
other labeled " $\longleftarrow$ ".

Prior to choosing an action in each round, each subject was told her own current position, the current position of the person with whom she was currently matched, and the number of subjects currently at each position. There were five possible current positions given by tick marks on a horizontal line:

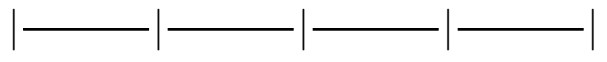

At the start of the first round, all subjects began in the middle position. That is, each subject's initial current position was

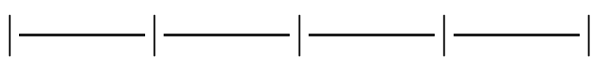

For round 2, if a subject had previously chosen " $\rightarrow$," then her current position would be shifted one tick mark to the right. Likewise, if in round 1 she had chosen " $\leftarrow$," her current position would be shifted one tick to the left. In all subsequent rounds, a similar application of this scheme determined all subjects' current positions with two exceptions. If a subject was at the rightmost tick mark and chose the action " $\rightarrow$," then she stayed at the rightmost tick mark. Similarly, if a subject was at the leftmost tick mark and chose action " $\leftarrow$," then she remained at the leftmost tick mark. Thus, a player's current position provided a signal about recent play. Figure 1 displays a typical first-round screen including current positions. For purposes of exposition, we shall number the five positions as follows:

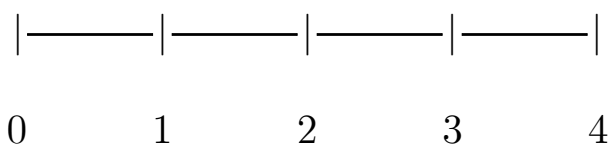

but no such delineation was made for the subjects participating in the experiment.

Following the selection of an action by all subjects in a given round, the payoff cell jointly selected by a subject and the matched opponent was illuminated on the subject's screen. Subjects then had to click their computer mice to continue to the next round. Subjects were kept continuously informed of their cumulative earnings throughout the session. At its conclusion, subjects were asked to fill out a post-experiment questionnaire explaining how they had played during the experiment and were paid their winnings privately. 
Early rounds of the experiment tended to be completed relatively slowly with more than a minute sometimes elapsing between the completion of successive rounds. ${ }^{5}$ By round 20, however, the speed with which decisions were being made increased substantially. The time elapsing between rounds was by then typically not more than about 15 seconds. This pace remained relatively constant for the remainder of the session; however, in some sessions play slowed down again in the last few rounds. During the early rounds, subjects appeared to be testing various strategies and generally getting a "feel" for the game. In later rounds, subjects seemed mostly to be just following whatever decision rules they had settled on. Occasionally there would be a round that was delayed by a subject seemingly deciding to re-evaluate her strategy.

\section{Treatments}

As discussed earlier, the treatments all involved a stage-game money payoff matrix of the form

\begin{tabular}{|c|c|}
\hline$z, z$ & $x-y, x+y$ \\
\hline$x+y, x-y$ & 0,0 \\
\hline
\end{tabular}

where $x>y \geq 0 .{ }^{6}$ When $z=0$, the game is a BOS variant, and when $z=x$ the game is an $\mathrm{HD}$ variant. $^{7}$

For all treatments we fixed $x$ at 20 cents, and we varied the amount $y$ across treatments within each game form (BOS or HD). Thus, we shall use the shorthand $\operatorname{BOS}\left(y^{\prime}\right)$ to denote a session where the BOS payoff matrix was used and the $y$ parameter was assigned the value $y^{\prime}$ (in cents); similarly for

\footnotetext{
${ }^{5}$ No play in a round was possible until all subjects had made choices in the preceding round.

${ }^{6} \mathrm{As}$ is customary, the first number in the cell is the payoff to the row player.

${ }^{7}$ To be precise, in our experimental implementation we added 5 cents to both payoffs in each cell of the matrix to guarantee that all subjects received at least $\$ 5$ for their time.
} 
$\mathrm{HD}\left(y^{\prime}\right){ }^{8}$ The following table summarizes the sessions we ran:

$\begin{array}{lc}\text { Treatment Name } & \text { \# of Sessions } \\ \operatorname{HD}(5) & 2 \\ \operatorname{HD}(10) & 4 \\ \operatorname{BOS}(0) & 2 \\ \operatorname{BOS}(5) & 6 \\ \operatorname{BOS}(10) & 2\end{array}$.

\section{Table 1: Summary of Treatments}

(We ran more sessions for $\mathrm{HD}(10)$ and $\operatorname{BOS}(5)$ because those treatments exhibited more cross-session variation.) For all of these treatments, the intuitive rule predicts the same behavior when subjects occupying different positions encounter one another. When the members of a matched pair occupy the same position, the prediction of the equilibrium of the theoretical model corresponding to the intuitive rule depends on both the assumed discount factor and the parameter $y$.

\section{Other Design Considerations}

Two other aspects of the experimental design are worth mentioning.

1. To keep the environment as simple as possible, we chose not to try to induce theoretically risk-neutral preferences through a binary lottery (e.g., Roth and Malouf (1979)) despite the fact that the games we study have mixed equilibria and more than two levels of payoffs. ${ }^{9}$

2. To control for the possibility that the labeling of actions might affect subject play, in half the sessions within each treatment we labeled the aggressive action " $\rightarrow$ " and in the other half " $\longleftarrow$ ". ${ }^{10}$ To test whether these labels had any effect on subject choices, we conducted a t-test of the proportions of strategically equivalent choices made under the two different labelings for each treatment. As an example, a player whose current position was 4 and who was matched with a 0 and chose $\rightarrow$ under one labeling would be deemed

\footnotetext{
${ }^{8}$ Thus Figure 1 is for $\mathrm{HD}(10)$.

${ }^{9} \mathrm{An}$ additional consideration in not employing this procedure is that its success in practice in inducing risk-neutral preferences has been mixed. Selten et al. (1995) report on experiments showing that making direct payments for actions may actually work better than binary lotteries in inducing risk-neutral behavior.

${ }^{10} \mathrm{In} \operatorname{BOS}(0)$, where the actions are equally aggressive, the statement does not apply.
} 
to be acting strategically equivalently to a 0 matched with a 4 choosing $\leftarrow$ under the reverse labeling. ${ }^{11}$ For a given treatment, we tested whether the proportion of right choices in the first case was equal to the proportion of left choices in the second for each strategically equivalent reputation pair. We find no significant labeling effect at the $5 \%$ level in the last 50 rounds of play for any pair in any treatment. As a consequence, in the following exposition we pool all sessions for a given treatment and rename the positions and choices in half the sessions so that " $\rightarrow$ " now always refers to the aggressive choice.

\section{Theory}

To provide some basis for judging the predictive power of the intuitive rule with respect to the experimental results, we adapt the Rosenthal-Landau model to our experimental setting.

For the one-shot game

\begin{tabular}{|c|c|c|}
\hline & $\leftarrow$ & $\rightarrow$ \\
\hline$\leftarrow$ & $z, z$ & $x-y, x+y$ \\
\hline$\rightarrow$ & $x+y, x-y$ & 0,0 \\
\hline
\end{tabular}

with 5 positions (labelled $0, \ldots, 4$ from left to right) and common discount factor $\beta$, a steady state equilibrium is a triple $(\pi, P, v)$ where

$\pi=\left(\pi_{0}, \pi_{1}, \pi_{2}, \pi_{3}, \pi_{4}\right)$ is the population's steady state relative-frequency distribution over the positions;

$P=$

$$
\left(\begin{array}{lll}
p_{00} & \cdots & p_{04} \\
\cdot & \cdots & \cdot \\
p_{40} & \cdots & p_{44}
\end{array}\right)
$$

where $p_{i j}$ is the probability that each individual assigns to the play of $\rightarrow$ when own position is $i$ and opponent's position is $j$; and

\footnotetext{
${ }^{11}$ Recall that the subjects were also kept apprised of the current population frequency distribution over positions. For this test, as for all others in this paper, we pooled the data across these different frequency distributions.
} 
$v=\left(v_{0}, v_{1}, v_{2}, v_{3}, v_{4}\right)$ is the vector of equilibrium present values.

The equilibrium conditions are:

$$
\begin{aligned}
& \pi_{0}=\pi_{1} \sum_{j} \pi_{j}\left(1-p_{1 j}\right)+\pi_{0} \sum_{j} \pi_{j}\left(1-p_{0 j}\right) \\
& \pi_{i}=\pi_{i+1} \sum_{j} \pi_{j}\left(1-p_{i+1, j}\right)+\pi_{i-1} \sum_{j} \pi_{j} p_{i-1, j}
\end{aligned}
$$

for $i=1,2,3$; and

$$
\pi_{4}=\pi_{4} \sum_{j} \pi_{j} p_{4 j}+\pi_{3} \sum_{j} \pi_{j} p_{3 j} .
$$

Adopting the conventions $4+1 \equiv 4$ and $0-1 \equiv 0$,

$$
\begin{array}{r}
v_{i}=\sum_{j} \pi_{j}\left[p_{i j}\left((x+y)\left(1-p_{j i}\right)+\beta v_{i+1}\right)\right. \\
\left.+\left(1-p_{i j}\right)\left((x-y) p_{j i}+z\left(1-p_{j i}\right)+\beta v_{i-1}\right)\right] \\
i=0, \ldots, 4 .
\end{array}
$$

Finally $\forall i, j, p_{i j}=1$ if

$$
(x+y)\left(1-p_{j i}\right)+\beta v_{i+1}>(x-y) p_{j i}+z\left(1-p_{j i}\right)+\beta v_{i-1} ;
$$

and $p_{i j}=0$ if

$$
(x+y)\left(1-p_{j i}\right)+\beta v_{i+1}<(x-y) p_{j i}+z\left(1-p_{j i}\right)+\beta v_{i-1} ;
$$

Of course, we must also have

$$
0 \leq p_{i j}, \pi_{i} \leq 1 \forall i, j \text {, and } \sum_{i} \pi_{i}=1 .
$$

(1)-(3) require that $\pi$ be an invariant distribution for the Markov chain generated by the given matching process and decision rule $P$. (4) defines $v$ as the vector of present values for a player finding herself in each position in the steady state, and the strict inequalities require that best responses be played with probability one. 
To illustrate, i.i.d. play of the symmetric one-shot equilibrium

$$
\left(\frac{x+y-z}{2 x-z}, \frac{x-y}{2 x-z}\right)
$$

generates a steady state equilibrium in which

$$
\begin{gathered}
p_{i j}=p \equiv \frac{x+y-z}{2 x-z} \forall i, j, \quad v_{i}=\frac{x^{2}-y^{2}}{(1-\beta)(2 x-z)} \forall i, \text { and } \\
\pi=\left(\sum_{i=0}^{4} p^{i}(1-p)^{4-i}\right)^{-1}\left((1-p)^{4}, p(1-p)^{3}, p^{2}(1-p)^{2}, p^{3}(1-p), p^{4}\right) .
\end{gathered}
$$

For BOS, $z=0$ and $y<x$; so $p \geq 1 / 2$, and the components of the $\pi-$ vector are nondecreasing. For HD, $z=x$, so $p=y / x$; and the $\pi$-distribution is nondecreasing or decreasing according to whether $y \geq x / 2$ or not. The value of $\beta$ plays no role in this steady state equilibrium since players foresee no future implications of their current actions.

As another illustration, the reverse intuitive rule is consistent with a steady state equilibrium for all the BOS and HD parametrizations of this experiment. Here the value of $\beta$ does enter the calculations, but as far as we have checked there seems to be a reverse-intuitive steady state equilibrium for each of the treatments for any $\beta \in[0,1) .{ }^{12}$ A typical $\pi$ - vector for such an equilibrium is $\cap$-shaped. For instance, for $\operatorname{BOS}(10)$ the steady state equilibrium when $\beta=.99$ has

$$
\begin{gathered}
p_{i j}= \begin{cases}0 & \text { if } i>j \\
1 & \text { if } i<j\end{cases} \\
\left(p_{00}, p_{11}, p_{22}, p_{33}, p_{44}\right) \cong(.50, .41, .49, .54, .58) \text { and } \\
\left(\pi_{0}, \pi_{1}, \pi_{2}, \pi_{3}, \pi_{4}\right) \cong(.26, .44, .26, .05, .00) .
\end{gathered}
$$

There are many other steady state equilibria for the models. Most of these, like the two above are grossly at odds with the experimental data. There are some variants of the intuitive rule, however, that generate steady state equilibria having qualitative characteristics similar to those of the equilibria that are generated by the intuitive rule. More on this later.

For the rest of this section, we describe steady state equilibria corresponding to the intuitive rule.

\footnotetext{
${ }^{12}$ This may be an artifact of the particular treatments chosen for the experiment. See Rosenthal and Landau (1979).
} 


\subsection{The BOS Model}

The intuitive rule has

$$
p_{i j}=\left\{\begin{array}{ll}
1 & \text { if } i>j \\
0 & \text { if } i<j
\end{array} .\right.
$$

To complete the specification of an equilibrium, we need to find $\left\{p_{i i}\right\}$ for which the $\pi$ and $v$ that solve (1)-(3) and (4) satisfy the best-response conditions. The tables below give the unique approximate $\left\{p_{i i}\right\}$ and $\pi$ values that do this for each of the three treatments for a variety of discount factors. In each case, the highest discount factor listed is close to the maximum discount factor for which the intuitive rule forms an equilibrium. ${ }^{13}$

$\begin{array}{llllll}\boldsymbol{\beta} & p_{00} & p_{11} & p_{22} & p_{33} & p_{44} \\ \mathbf{4} & .528 & .535 & .500 & .465 & .472 \\ . \mathbf{5} & .536 & .547 & .500 & .453 & .464 \\ \mathbf{. 6} & .545 & .561 & .500 & .439 & .455 \\ . \mathbf{7 5} & .561 & .585 & .500 & .415 & .439 \\ . \mathbf{8 5} & .573 & .604 & .500 & .396 & .427 \\ . \mathbf{9 9 5} & .594 & .638 & .500 & .362 & .406\end{array}$

Table 2: Steady State Play of $\rightarrow$ in BOS(0) from Equal Positions

$\begin{array}{llllll}\boldsymbol{\beta} & p_{00} & p_{11} & p_{22} & p_{33} & p_{44} \\ . \mathbf{4} & .677 & .707 & .669 & .635 & .621 \\ .5 & .695 & .740 & .693 & .644 & .622 \\ . \mathbf{6} & .715 & .783 & .730 & .662 & .625 \\ . \mathbf{7 5} & .755 & .880 & .829 & .722 & .642 \\ . \mathbf{8 5} & .794 & .987 & .961 & .818 & .675\end{array}$

Table 3: Steady State Play of $\rightarrow$ in BOS(5) from Equal Positions

\footnotetext{
${ }^{13}$ The intuitive rule forms a steady state equilibrium for every nonnegative discount factor between zero and the maximum for each treatment.
} 


$$
\begin{array}{llllll}
\boldsymbol{\beta} & p_{00} & p_{11} & p_{22} & p_{33} & p_{44} \\
\mathbf{. 4} & .826 & .877 & .833 & .797 & .765 \\
. \mathbf{5} & .850 & .928 & .879 & .825 & .774 \\
\mathbf{. 6} & .879 & .993 & .944 & .868 & .789
\end{array}
$$

Table 4: Steady State Play of $\rightarrow$ in BOS(10) from Equal Positions

Notice that for those cases where the same discount factor appears in more than one of the tables above, holding that discount factor and the position $i$ fixed and letting the $y$ parameter increase always results in an increased value for $p_{i i}$.

$\begin{array}{llllll}\boldsymbol{\beta} & \boldsymbol{\pi}_{0} & \boldsymbol{\pi}_{1} & \boldsymbol{\pi}_{2} & \boldsymbol{\pi}_{3} & \boldsymbol{\pi}_{4} \\ \mathbf{. 4} & .348 & .108 & .088 & .108 & .348 \\ \mathbf{. 5} & .347 & .109 & .088 & .109 & .347 \\ \mathbf{. 6} & .346 & .110 & .089 & .110 & .346 \\ \mathbf{. 7 5} & .343 & .112 & .091 & .112 & .343 \\ \mathbf{8 5} & .341 & .113 & .092 & .113 & .341 \\ \mathbf{. 9 9 5} & .338 & .115 & .095 & .115 & .338\end{array}$

Table 5: Steady State Position Frequencies in BOS(0)

$$
\begin{array}{llllll}
\boldsymbol{\beta} & \boldsymbol{\pi}_{0} & \boldsymbol{\pi}_{1} & \boldsymbol{\pi}_{2} & \boldsymbol{\pi}_{3} & \boldsymbol{\pi}_{4} \\
. \mathbf{4} & .316 & .112 & .086 & .099 & .387 \\
. \mathbf{5} & .313 & .113 & .087 & .099 & .388 \\
. \mathbf{6} & .309 & .113 & .087 & .100 & .391 \\
\mathbf{. 7 5} & .300 & .113 & .088 & .100 & .400 \\
. \mathbf{8 5} & .290 & .112 & .087 & .100 & .414
\end{array}
$$

Table 6: Steady State Position Frequencies in BOS(5) 


$\begin{array}{llllll}\boldsymbol{\beta} & \boldsymbol{\pi}_{0} & \boldsymbol{\pi}_{1} & \boldsymbol{\pi}_{2} & \boldsymbol{\pi}_{3} & \boldsymbol{\pi}_{4} \\ . \mathbf{4} & .288 & .111 & .080 & .082 & .438 \\ . \mathbf{5} & .283 & .111 & .080 & .082 & .443 \\ \mathbf{6} & .278 & .111 & .080 & .080 & .452\end{array}$

\section{Table 7: Steady State Position Frequencies in BOS(10)}

Notice that in each of the three treatments, the $\pi$ distribution is $\cup$-shaped and not very sensitive to changes in the discount factor.

\subsection{The HD Model}

Approximate values of $\left\{p_{i i}\right\}$ and $\pi$ which together with the $\left\{p_{i j}\right\}_{j \neq i}$ of the intuitive rule generate steady state for various $\beta$-values for the HD treatments are given in the tables below.

$\begin{array}{llllll}\boldsymbol{\beta} & p_{00} & p_{11} & p_{22} & p_{33} & p_{44} \\ . \mathbf{5} & .359 & .435 & .390 & .378 & .314 \\ \mathbf{. 6} & .396 & .516 & .472 & .442 & .340 \\ \mathbf{. 7 5} & .473 & .708 & .697 & .622 & .410 \\ .8 & .506 & .802 & .820 & .725 & .451 \\ \mathbf{8 5} & .544 & .915 & .979 & .865 & .507\end{array}$

Table 8: Steady State Play of $\rightarrow$ in $\mathrm{HD}(5)$ from Equal Positions

$\begin{array}{llllll}\boldsymbol{\beta} & p_{00} & p_{11} & p_{22} & p_{33} & p_{44} \\ . \mathbf{5} & .688 & .844 & .781 & .744 & .618 \\ \mathbf{6} & .740 & .970 & .924 & .859 & .665\end{array}$

Table 9: Steady State Play of $\rightarrow$ in $\mathrm{HD}(10)$ from Equal Positions 
Again (at least for the one case of a discount factor in common) each $p_{i i}$ value increases in $y$.

$\begin{array}{llllll}\boldsymbol{\beta} & \boldsymbol{\pi}_{0} & \boldsymbol{\pi}_{1} & \boldsymbol{\pi}_{2} & \boldsymbol{\pi}_{3} & \boldsymbol{\pi}_{4} \\ . \mathbf{5} & .389 & .095 & .085 & .113 & .318 \\ . \mathbf{6} & .376 & .100 & .086 & .114 & .325 \\ . \mathbf{7 5} & .351 & .101 & .088 & .115 & .346 \\ . \mathbf{8} & .340 & .101 & .087 & .115 & .357 \\ \mathbf{. 8 5} & .327 & .100 & .0860 & .113 & .374\end{array}$

Table 10: Steady State Position Frequencies in HD(5)

$\begin{array}{llllll}\boldsymbol{\beta} & \boldsymbol{\pi}_{0} & \boldsymbol{\pi}_{1} & \boldsymbol{\pi}_{2} & \boldsymbol{\pi}_{3} & \boldsymbol{\pi}_{4} \\ .5 & .310 & .110 & .087 & .101 & .392 \\ .6 & .297 & .109 & .086 & .098 & .410\end{array}$

Table 11: Steady State Position Frequencies in $\operatorname{HD}(10)$

Here the $\pi$ distribution is more sensitive to changes in $\beta$.

\section{Results}

\subsection{Preliminary Analysis}

In this section, we examine whether observed behavior (in the last 50 rounds) appears qualitatively consistent with the intuitive rule overall. First, we present summary statistics on the frequency of choices that accord with the intuitive rule when matched positions are unequal.

$\begin{array}{cc}\text { Treatment } & \text { \% Compliant } \\ \text { BOS }(0) & 97.8 \\ \text { BOS(5) } & 90.8 \\ \text { BOS(10) } & 81.4 \\ \text { HD }(5) & 91.0 \\ \text { HD }(10) & 72.9\end{array}$

All treatments $\quad 85.9$

Table 12: Compliance with Intuitive Rule 
As Table 12 makes clear, in all treatments, levels of compliance with the intuitive rule are considerably in excess of what might occur by chance. Furthermore, the compliance rates within each game form are monotonically decreasing in $y$. This may be formalized by testing for equality in mean compliance rates across treatments. Table 13 below presents t-statistics for pairwise comparisons of compliance rates. In all cases, differences in the $y$ parameter are significant at the $5 \%$ level.

$\begin{array}{cc}\text { Treatment } & \text { t-statistic } \\ \text { BOS }(0) \text { vs BOS }(5) & 6.88 \\ \text { BOS }(5) \text { vs BOS }(10) & 7.35 \\ \text { HD }(5) \text { vs } \operatorname{HD}(10) & 10.87\end{array}$

Table 13: Differences in $y$ parameter

Interestingly, if we hold fixed the value of the $y$ parameter and compare compliance rates for different $z$ values, we find that there is a significant difference in the compliance rates of $\operatorname{BOS}(10)$ as compared to $\mathrm{HD}(10)(t=$ 4.59), but no significant difference between $\operatorname{BOS}(5)$ and $\operatorname{HD}(5)(t=0.18)$. Thus, compliance rates seem to be more sensitive to variations in $y$ than to variations in $z$.

To see roughly how much information about current positions affects play, it is useful to compare our compliance rates to the results of Cooper, et al. (1994). They report results of the last eleven rounds of three round-robin experiments where each session consisted of eleven players playing BOS(10). Out of 165 plays, only $41 \%$ resulted in coordinated off-diagonal outcomes. Cooper, et al. do not report choice data by individuals, but their aggregate statistics are roughly consistent with the predictions of i.i.d. draws from the symmetric mixed strategy equilibrium of the game above in which each player randomizes with probability $3 / 4$ on the aggressive action. To see the comparison another way, in our BOS(10) treatment $58.5 \%$ of the joint choices overall and $65.6 \%$ of the joint choices from unequal positions resulted in offdiagonal outcomes. These numbers are considerably higher than $41 \%$. This comparison suggests that information about current positions is probably playing a large role in facilitating coordination in our experiment. ${ }^{14}$

\footnotetext{
${ }^{14}$ Cooper et al. also report on a treatment in which prior to each play one of the players was permitted (but not forced) to announce the listed name of one of the strategies, with
} 
To get a feel for the evolution of play in our experiment, Table 14 presents compliance percentages (from unequal positions) for specific rounds. Here we see that while the intuitive custom is, to some extent, learned by the subjects; nonetheless, a considerable proportion of early moves is already consistent with the intuitive rule. This suggests that the intuitive rule might indeed be intuitive or instinctive for many subjects.

\begin{tabular}{cccc} 
Treatment & Rd. 2 & Rd. 51 & Rd 100 \\
HD $(5)$ & 100 & 100 & 100 \\
HD $(10)$ & 63.6 & 68.8 & 78.1 \\
BOS $(0)$ & 83.3 & 100 & 100 \\
BOS $(5)$ & 63.3 & 86.5 & 92.6 \\
BOS $(10)$ & 88.9 & 94.4 & 100 \\
All treatments & 71.8 & 87.3 & 91.7 \\
\multicolumn{4}{c}{ Table 14: Compliance Over Time }
\end{tabular}

Several aspects of Table 14 are worth highlighting. First, more than $2 / 3$ of subject choices were already consistent with the intuitive custom in Round 2 of the experiment. ${ }^{15}$ By round 51, overall compliance rates increased farther to around $87 \%$. In two treatments, $\operatorname{HD}(5)$ and $\operatorname{BOS}(0)$, the data were perfectly consistent with the intuitive rule in this round. And, over the last 50 rounds of the game, there was only a slight upward trend in compliance rates. A feeling for the stationarity of compliance with the intuitive rule over the last 50 rounds may also be obtained from Figures 2-7, which depict average compliance rates across treatments over all rounds. As Figures 2-7 also illustrate, there are no obvious endgame effects in the compliance rates.

The theoretical model hypothesizes that individuals are playing stationary strategies in an infinitely repeated game. A necessary condition for the data to be consistent with this hypothesis is that the empirical frequency distributions of current positions be constant. To test this, we divided the

no previous discussion of what signal such announcements might convey. In 157 of the 165 observations, the designated player announced the name of his aggressive strategy; and in 154 of these the pair successfully coordinated on the favored outcome of the announcer. Evidently, the subjects had little difficulty in appreciating and responding to this particular desymmetrizing opportunity.

${ }^{15}$ Since all subjects begin in the same current position, round 1 provides no compliance data. 
observations of current positions into two groups: rounds 51-75 and rounds 76-100. The theoretical prediction is that the empirical frequency of current positions should be the same in rounds 51-75 as in rounds 76-100. Table 15 below reports p-values obtained from Fisher exact tests of this null hypothesis. As the table shows, the null hypothesis is rejected at the $5 \%$ significance level in one of the $\mathrm{HD}(10)$ sessions (session 4) and both of the BOS(0) sessions, but not in the remaining 13 sessions. In the sessions where the null hypothesis is rejected, much of the variation appears to occur in rounds 5160 ; thus, if instead we examine only the last 40 rounds divided evenly into two groups (third column of Table 15), we find that the null hypothesis is not rejected at the $5 \%$ level in any of the previously rejected sessions. ${ }^{16}$

\begin{tabular}{|c|c|c|c|}
\hline \multirow[b]{2}{*}{ Session } & \multirow[b]{2}{*}{ Treatment } & \multicolumn{2}{|c|}{ p-values } \\
\hline & & Rds 51-100 & Rds 61-100 \\
\hline 1 & $\mathrm{HD}(10)$ & .578 & .184 \\
\hline 2 & $\mathrm{HD}(10)$ & .288 & .200 \\
\hline 3 & $\mathrm{HD}(10)$ & .080 & .507 \\
\hline 4 & $\mathrm{HD}(10)$ & $.008^{*}$ & .147 \\
\hline 5 & BOS(10) & .257 & .163 \\
\hline 6 & BOS(10) & .349 & .979 \\
\hline 7 & $\operatorname{BOS}(5)$ & .943 & .976 \\
\hline 8 & $\operatorname{BOS}(5)$ & .127 & .837 \\
\hline 9 & $\operatorname{BOS}(5)$ & .127 & .278 \\
\hline 10 & $\operatorname{BOS}(5)$ & .694 & .583 \\
\hline 11 & $\operatorname{BOS}(5)$ & .916 & .382 \\
\hline 12 & $\operatorname{BOS}(5)$ & .692 & .568 \\
\hline 13 & $\mathrm{HD}(5)$ & .119 & $.034^{*}$ \\
\hline 14 & $\mathrm{HD}(5)$ & .777 & .446 \\
\hline 15 & $\operatorname{BOS}(0)$ & $.049^{*}$ & .437 \\
\hline 16 & $\operatorname{BOS}(0)$ & $.010^{*}$ & .279 \\
\hline
\end{tabular}

\section{Table 15: Fisher Exact Tests of Stationarity}

To summarize, the introduction of current position information clearly increases the frequency with which coordination is achieved relative to a

\footnotetext{
${ }^{16}$ We reject at the $5 \%$ level in only one session, a different one, using this methodology.
} 
similar experiment where this information is absent. Moreover, the form this coordinating behavior takes appears to be qualitatively consistent with the predictions of the intuitive custom and relatively stationary for the last half of each session. To see how well the data fit the more detailed predictions of the theoretical model, we shall have to look more closely at the data for each treatment.

\subsection{BOS Results}

We turn first to more detailed analyses of the results from the BOS treatments.

\section{$\operatorname{BOS}(0)$}

In $\operatorname{BOS}(0)$, there is no difference in the aggressiveness of the two actions. Here, current positions merely serves as a signal of the tendency of a particular player to be choosing the action labelled $\rightarrow$ rather than the one labelled $\leftarrow$. Perhaps as a consequence of this, the compliance rates were highest for this treatment. To see in more detail the patterns of compliance, it is useful to examine the proportion of plays where the $\rightarrow$ action was chosen as a function of the current position pairs. This information is presented for the last 50 rounds of the two $\operatorname{BOS}(0)$ sessions in Table 16, where the entries are the percentages of instances of $\rightarrow$ play from each position pair and the numbers in parentheses are the totals of observed instances of the respective position pairs. (Thus, for instance, the numbers in row 0 column 4 indicate that $99.4 \%$ (i.e., 172) of the 173 instances of subjects in position 4 facing an opponent in position 0 played $\rightarrow$.)

\begin{tabular}{lllllll} 
& \multicolumn{6}{c}{ Player's Own Current Position } \\
& & $\mathbf{0}$ & $\mathbf{1}$ & $\mathbf{2}$ & $\mathbf{3}$ & $\mathbf{4}$ \\
& $\mathbf{0}$ & $50.1(126)$ & $97.6(42)$ & $100(35)$ & $100(44)$ & $99.4(173)$ \\
Opponent's & $\mathbf{1}$ & $11.9(42)$ & $50.0(8)$ & $100(10)$ & $100(9)$ & $100(43)$ \\
Current & $\mathbf{2}$ & $0.0(35)$ & $0.0(10)$ & $50.0(6)$ & $100(8)$ & $100(33)$ \\
Position & $\mathbf{3}$ & $0.0(44)$ & $0.0(9)$ & $0.0(8)$ & $75.0(4)$ & $86.3(51)$ \\
& $\mathbf{4}$ & $0.6(173)$ & $0.0(43)$ & $0.0(33)$ & $9.8(51)$ & $61.2(160)$
\end{tabular}

Table 16: Play of $\rightarrow$ in $\operatorname{BOS}(0)$ 
In the case of players in unequal positions, as already noted, the intuitive prediction does remarkably well. Indeed, out of 896 instances of choices in unequal current positions, $876(97.8 \%)$ were in compliance with the intuitive rule. Interestingly, of the 20 noncompliant cases, all but two were from $(3,4)$ or $(0,1)$ pairings. Moreover, 7 of the 20 instances of non-compliance were the actions of a single subject.

In the case of players with equal current positions (the diagonal cells in Table 16), the equilibrium prediction of the model does less well, no matter what discount factor is assumed. There are too few observations to make much of the comparison with the theoretical calculations of $p_{11}, p_{22}$, and $p_{33}$ from Table 2, but the extreme diagonal entries of $50.1 \%$ and $64.2 \%$ are lower and higher, respectively, than all the numbers in the $p_{00}$ and $p_{44}$ columns, respectively, for $\operatorname{BOS}(0)$ in Table 2 . Indeed, the equilibrium predictions in these cells are evidently worse than the predictions of the one-shot mixed Nash equilibrium of the game, which entails $\rightarrow$ play with probability .5 . However, a t-test of the null hypothesis that the probability of right play is .5 when players of equal current positions are matched shows that even this hypothesis is rejected at conventional significance levels $(t=2.1931)$. This rejection is largely accounted for by the fact that $(4,4)$ matches result in too much $\rightarrow$ play.

Table 17 reports the empirical frequencies of the positions for the last 50 rounds of $\operatorname{BOS}(0)$.

\begin{tabular}{cccccc} 
& \multicolumn{5}{c}{ Position } \\
Frequency & $\mathbf{0}$ & $\mathbf{1}$ & $\mathbf{2}$ & $\mathbf{3}$ & $\mathbf{4}$ \\
& .350 & .093 & .077 & .097 & .383
\end{tabular}

\section{Table 17: Position Frequencies in $\operatorname{BOS}(0)$}

Notice that these empirical frequencies match up fairly closely with the predictions of the theoretical model in Table 5 for all discount factors.

\section{$\operatorname{BOS}(5)$}

Table 18 presents the $\rightarrow$ play data for the six BOS(5) sessions analogously to Table 16. 


\begin{tabular}{lllllll} 
& & \multicolumn{5}{c}{ Player's Own Current Position } \\
& & $\mathbf{0}$ & $\mathbf{1}$ & $\mathbf{2}$ & $\mathbf{3}$ & $\mathbf{4}$ \\
& $\mathbf{0}$ & $50.4(250)$ & $87.9(91)$ & $91.4(70)$ & $94.5(110)$ & $97.3(510)$ \\
Opponent's & $\mathbf{1}$ & $19.8(91)$ & $66.7(18)$ & $87.0(23)$ & $85.7(35)$ & $98.5(130)$ \\
Current & $\mathbf{2}$ & $8.6(70)$ & $39.1(23)$ & $78.6(14)$ & $96.0(25)$ & $96.7(120)$ \\
Position & $\mathbf{3}$ & $6.4(110)$ & $37.1(35)$ & $32.0(25)$ & $76.2(42)$ & $93.4(168)$ \\
& $\mathbf{4}$ & $2.2(510)$ & $10.8(130)$ & $14.2(120)$ & $41.1(168)$ & $67.8(712)$
\end{tabular}

Table 18: Play of $\rightarrow$ in $\operatorname{BOS}(5)$

Overall in the $\operatorname{BOS}(5)$ sessions, the compliance rate was $90.8 \%$ from unequal positions. Of the 235 instances of non-compliance, 80 were from $(3,4)$ matches. The majority of these instances of noncompliance were, in turn, cases where in the previous period the subject had been in the extreme state 4 and played the nonaggressive strategy. ${ }^{17}$ Further, 93 of the 235 instances of non-compliance occurred in one session (\#10). Overall, the compliance rates for BOS(5) seem high enough that any simple hypothesis about subjects play must involve something very close to the intuitive rule for the situations when the matched subjects are in unequal positions. As before, the vast majority of the 72 subjects in these sessions played either perfectly or almost perfectly in accord with the prescriptions of the intuitive rule. Specifically, only 10 subjects out of 72 had compliance rates below $85 \%$.

From the equal-position pairs $(0,0)$ and $(1,1), \rightarrow$ was chosen far too infrequently relative to the equilibrium calculations of Table 3. Equilibrium predictions for $(2,2),(3,3)$, and $(4,4)$ for discount factors around .75 are more in line with the observed data. The one-shot mixed strategy equilibrium for this game is for both players to play $\rightarrow$ with probability $62.5 \%$, and the fact that the proportion of $\rightarrow$ play from these three position pairs exceeded this level appears to reflect reputation-based reasoning.

Table 19 reports the empirical frequencies of each position observed in the last 50 rounds of the $\operatorname{BOS}(5)$ sessions. It seems closest to the $\mathrm{BOS}(5)$ $\pi$ - distribution for the discount factor .85 in Table 2 .

\footnotetext{
${ }^{17}$ This pattern recurs in other treatments. See Section 5 for attempts to explain it.
} 


\section{Position

$\begin{array}{cccccc} & \mathbf{0} & \mathbf{1} & \mathbf{2} & \mathbf{3} & \mathbf{4} \\ \text { Frequency } & .286 & .083 & .070 & .106 & .456\end{array}$

Table 19: Position Frequencies in BOS(5)

There is a pronounced rightward bias in both play and position frequencies in the BOS(5) data. This is due to two effects: (1) a bias toward the aggressive strategy when a player and the matched opponent are in the same position (which is qualitatively consistent with the predictions of the theoretical model in Section 2), and (2) a bias toward the aggressive strategy among the instances in which the prescription of the intuitive rule was not followed (about which the theoretical model is silent).

\section{BOS(10)}

Table 20 presents the $\rightarrow$ play data for the two BOS(10) sessions. From unequal positions, the compliance rate was $81.4 \%$. As in the $\operatorname{BOS}(5)$ sessions, a significant proportion (60 out of 145) of the instances of noncompliance occurred in $(3,4)$ matches. Excluding these pairings raises the compliance rate in $\operatorname{BOS}(10)$ sessions to $85.8 \%$.

\begin{tabular}{llllllll} 
& & \multicolumn{5}{c}{ Player's Own Current Position } \\
& & $\mathbf{0}$ & $\mathbf{1}$ & $\mathbf{2}$ & $\mathbf{3}$ & $\mathbf{4}$ \\
& $\mathbf{0}$ & $59.4(32)$ & $92.9(14)$ & $93.8(16)$ & $90.9(33)$ & $93.6(126)$ \\
Opponent's & $\mathbf{1}$ & $28.6(14)$ & $-(0)$ & $75.0(8)$ & $100(8)$ & $91.9(37)$ \\
Current & $\mathbf{2}$ & $12.5(16)$ & $50.0(8)$ & $50.0(2)$ & $81.3(16)$ & $97.6(42)$ \\
Position & $\mathbf{3}$ & $6.1(33)$ & $50.0(8)$ & $50.0(16)$ & $86.7(30)$ & $85.4(89)$ \\
& $\mathbf{4}$ & $5.6(126)$ & $29.7(37)$ & $50.0(42)$ & $52.8(89)$ & $73.4(358)$
\end{tabular}

Table 20: Play of $\rightarrow$ in $\operatorname{BOS}(10)$

For players with equal current positions, most of the observations are $(4,4)$ pairings, and the predictions from Table 4 are not too far off here (though they are for $p_{00}$, where far too little $\rightarrow$ play is observed relative to the predictions of the theoretical model). In addition, because of the $(4,4)$ data, we cannot reject the null hypothesis that equal-position players are simply choosing $\rightarrow$ with the same probability as the one-shot mixed strategy equilibrium (probability $=.75, t=-0.8235$ ). It is hard to reconcile either of 
these with the large number of noncompliant choices when 2's and 3's were matched with 4's.

The position frequencies are listed in Table 21.

$$
\begin{array}{cccccc} 
& \multicolumn{5}{c}{\text { Position }} \\
\text { Frequency } & .184 & \mathbf{1} & \mathbf{2} & \mathbf{3} & \mathbf{4} \\
& .056 & .070 & .147 & .543
\end{array}
$$

\section{Table 21: Position Frequencies in BOS(10)}

Table 21 reveals that as in $\operatorname{BOS}(5)$, there is a pronounced rightward bias in play even relative to the $\pi$ - distributions for $\operatorname{BOS}(10)$. This is evidently mostly due to the aforementioned noncompliant choices of 2's and 3's matched with 4's.

\subsection{HD Results}

We now turn to a detailed analysis of the results from the HD treatments.

HD(5)

Table 22 presents the $\rightarrow$ play data for the two $\mathrm{HD}(5)$ sessions. From

\begin{tabular}{|c|c|c|c|c|c|c|}
\hline & \multicolumn{5}{|c|}{ Player's Own Current Position } \\
\hline & & $\mathbf{0}$ & 1 & 2 & 3 & 4 \\
\hline & $\mathbf{0}$ & $27.1(236)$ & $58.5(53)$ & $96.9(32)$ & $93.3(60)$ & $98.1(156)$ \\
\hline Opponent's & 1 & $7.5(53)$ & $16.7(6)$ & $75.0(8)$ & $87.5(8)$ & $94.9(39)$ \\
\hline Current & 2 & $9.4(32)$ & $12.5(8)$ & $50.0(8)$ & $71.4(7)$ & $93.1(29)$ \\
\hline Position & 3 & $1.7(60)$ & $0.0(8)$ & $0.0(7)$ & $31.3(16)$ & $81.3(32)$ \\
\hline & 4 & $3.8(156)$ & $12.8(39)$ & $24.1(29)$ & $12.5(32)$ & $27.9(26)$ \\
\hline
\end{tabular}
unequal positions, the compliance rate was $91.0 \%$.

\section{Table 22: Play of $\rightarrow$ in $\mathrm{HD}(5)$}

For this treatment, the bulk of non-compliant play did not come from $(3,4)$ pairings: only 10 of the 76 instances of non-compliance were from $(3,4)$ matches. Instead, the largest portion (26) of the 76 non-compliant plays came from $(1,0)$ matches. This change from the BOS pattern is due, no doubt, at least in part, to the fact that in $\mathrm{HD}$ failing to coordinate by playing $\leftarrow$ is less costly than failing to coordinate by playing $\rightarrow$. Although the 
equilibrium calculations take account of this effect, our subjects evidently overcompensated: as evidenced by much-too-low (relative to equilibrium) incidence of $\rightarrow$ play from equal positions. Notice that in $\operatorname{HD}(5)$ sessions, the mixed-strategy equilibrium prediction of the one-shot game is that $\rightarrow$ will arise with probability .25 . In a t-test, we fail to reject the null hypothesis that the proportion of $\rightarrow$ choices from equal positions is equal to the one-shot mixed strategy probability $(t=1.1875)$ at conventional significance levels.

The position frequencies are listed in Table 23.

\section{Position

$\begin{array}{cccccc} & \mathbf{0} & \mathbf{1} & \mathbf{2} & \mathbf{3} & \mathbf{4} \\ \text { Frequency } & .448 & .095 & .070 & .103 & .285\end{array}$

Table 23: Position Frequencies in $\mathrm{HD}(5)$

These empirical frequencies are closest to the theoretical $\pi$ - distribution for relatively low discount factors, but, unlike the BOS treatments, the empirical position frequencies in $\mathrm{HD}(5)$ reflect a leftward bias even relative to the leftbiased predictions of the theoretical model for low discount factors. Even for discount rates as low as $\beta=.5$, there are more 0 types and fewer 4 types than is predicted by the equilibrium corresponding to the intuitive rule. This may all be accounted for by the bias toward $\leftarrow$ play in equal-position matches, but the underlying cause may be other-regarding preferences, such as a taste for fairness, that might encourage subjects to try to coordinate on $(\leftarrow, \leftarrow)$ play; thus achieving an equal split of the available earnings in a given round.

\section{$\operatorname{HD}(10)$}

Finally, we come to the four $\operatorname{HD}(10)$ sessions; Table 24 presents the $\rightarrow$ play data. From unequal positions the overall compliance rate was only $72.9 \%$.

\begin{tabular}{lllllll}
\multicolumn{7}{c}{ Player's Own Current Position } \\
& & $\mathbf{0}$ & $\mathbf{1}$ & $\mathbf{2}$ & $\mathbf{3}$ & $\mathbf{4}$ \\
Opponent's & $\mathbf{0}$ & $27.4(190)$ & $56.7(97)$ & $62.5(96)$ & $75.0(100)$ & $90.8(238)$ \\
Current & $\mathbf{2}$ & $20.5(97)$ & $37.0(46)$ & $40.0(30)$ & $62.2(37)$ & $86.1(101)$ \\
Position & $\mathbf{3}$ & $31.0(100)$ & $33.3(30)$ & $35.3(34)$ & $56.0(25)$ & $85.7(84)$ \\
& $\mathbf{4}$ & $20.6(238)$ & $48.5(101)$ & $40.0(25)$ & $71.4(28)$ & $74.5(106)$ \\
& \multicolumn{7}{c}{ Table 24: Play of $\rightarrow$ in HD(10) } &
\end{tabular}


As the table makes clear, the predictions of the intuitive-rule equilibrium did not fare at all well when players in positions 1-3 were matched with one another. Players with current position 0 and 4 played in a manner that is more consistent with the intuitive rule. If we pool all of the observations of choices by players from equal positions and compare this to the proportion of $\rightarrow$ choices predicted in the one-shot mixed-strategy equilibrium (.5), we again cannot reject the null hypothesis at conventional significance levels. However, this is largely due to the fact that the prevalence of $\leftarrow$ play from positions $(0,0),(1,1)$ and $(2,2)$ is being offset by the prevalence of $\rightarrow$ play by $(3,3)$ and $(4,4)$ pairs rather than the fact that players with equal current positions are playing the one-shot mixed strategy.

The position frequencies are listed in Table 25.

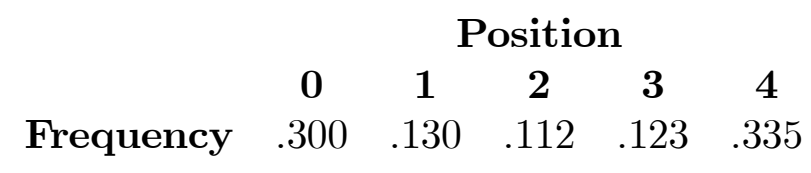

Table 25: Position Frequencies in $\mathrm{HD}(10)$

Relative to the equilibrium calculations, position 4 is underrepresented. This leftward bias appears less pronounced than in the $\operatorname{HD}(5)$ sessions however.

\section{Discussion}

The experimental results appear to us to support the following:

1. Subjects attempted to use position pairs as coordinating devices eventually in all sessions, and the intuitive rule, or something close to it, was the custom that invariably arose.

2. Adherence to the rule decreased in the $y$ - parameter for each game form.

3. Aggregate play in each session settled down to the semblance of a stationary pattern by Round 50 .

4. Adherence to the play predictions of the steady state equilibrium corresponding to the intuitive rule was better from unequal position pairs than from equal position pairs.

We repeat our earlier caveat that little of this can be established statistically both because the intuitive rule admits no variance from unequal 
position pairs and because we have not run nearly enough sessions of any one treatment. ${ }^{18}$ Furthermore, it could be that behavior depends in unexpected ways on the specific treatment variables that we kept fixed. There is an unlimited number of variations that would be interesting to try: total number of rounds fixed $\neq 100$, random number of rounds not told to subjects in advance, diminishing payoff magnitudes over time to simulate discounting, different $x$ and $y$ parameters, number of positions $\neq 5$, current position distribution hidden from subjects, different monetary scale, different subject pool, etc. It is impossible to know whether any of these variations would produce dramatically different patterns.

However, in assessing the predictive power of the intuitive rule, certain of these variations would be less useful. For instance, reducing the number of positions to three would lead to a greater proportion of tied reputational outcomes. This would lead to substantially fewer instances where reputational effects offer desymmetrizing opportunities and make it more difficult to distinguish what rule, if any, subjects were using. Adding additional positions beyond 5, in contrast, increases the complexity of the experiment and likely leads to a longer period before behavior "settles down." Thus, the experimental design decision to use five current positions represents an attempt to balance simplicity with the need to generate a substantial number of desymmetrizing opportunities in current positions.

Assuming that we are seeing a pattern that would be robust to such variations, does it occur because subjects are conditioned to the intuitive rule from their ordinary life experiences? Assuming yes to this, is the intuitive rule so pervasive in the real world because of the coordination role analogous to what is isolated in this experiment, or could it be that it serves a variety of functions of which this is only one? It is impossible to know, but we suspect the coordinating role is at least one of its important functions if not its most important function. This leaves the question of why the intuitive rule is so prevalent when there are so many other possible coordinating rules. Despite the suggestions in Section 1, we do not really know.

Although there are obvious mismatches between the assumptions of the model in Section 4 and the experiment, the model does reasonably well at matching up with the empirical $\pi$ - distribution, although less well for the $\left\{p_{i i}\right\}$. This should perhaps not be too surprising given the number of exper-

\footnotetext{
${ }^{18}$ One could argue that because of the degree of interaction among subjects in a given session, one session might properly be treated as a single observation.
} 
iments on one-shot games in which subjects do not appear to play mixed strategies in equilibrium proportions. ${ }^{19}$

Of course, with subjects being paid only at the end of the experiment, it is not obvious why they should discount at all, let alone geometrically or with particular common discount factors. While we cannot defend as realistic the specific form of the discounting assumed in the model, we suspect that subjects do implicitly trade off present against future winnings as they make choices in each round. And, perhaps because they are accustomed to accept unthinkingly the advice about the relative value of a bird in the hand, or because they are risk averse and perceive less risk in the results of play in the current round, or for some other reason, for the most part they seem to discount the future. Evidence for this is, somewhat circularly, based on the observation that they mostly play according to the intuitive rule. Consider for instance, a single individual in a large population of people playing BOS with positive $y$ according to the intuitive equilibrium for a particular common discount factor. Since $v_{4}$ is the highest equilibrium present value, if the individual's discount factor were higher than that of the rest of the population or if the individual didn't discount at all, the individual would not be indifferent in the equal-position matches, would eventually get to position 4, and would always play to the right. A small proportion of our subjects did seem to behave this way, but only a small proportion. If most subjects did, the average frequency of position 4 would be high and so would the instances of $(\rightarrow, \rightarrow)$ play (which gives a hint about why existence fails in the model when the discount factor is too high relative to $y$ ). This also suggests why the theoretical model may not predict too badly if most subjects discount the future, even if they do not all have a common discount factor or do not discount geometrically. Because of the anonymity and the relatively large numbers of subjects in each session, what is important is that there is enough discounting going on in the aggregate. Even if nobody is exactly indifferent at any equal-position match, if there is enough heterogeneity each individual faces an environment that looks as though all opponents are behaving similarly, though not deterministically. And it is only the conjectures individuals have about their aggregate environment that matter in their respective optimization problems. In other words, there are analogues of steady state equilibria for repeated anonymous matching games played by heterogeneous popula-

\footnotetext{
${ }^{19}$ See, e.g., Brown and Rosenthal (1990), Rapoport and Boebel (1992), Mookherjee and Sopher (1994), Shachat (1996).
} 
tions, but, when they exist, their aggregate behavior is always akin to that of equilibria of some homogeneous population model.

Similar observations can be used to rationalize the use of an infinitepopulation model when the population size is in fact finite. For some large enough number of subjects (we hope 12 is large enough), with anonymity reasonable individuals should be playing as if there is some undifferentiated mass of opponents.

As for the assumption that the stage-game money payoffs are von-NeumannMorgenstern utilities, since the money payoff in any round is small compared with total money payoff, risk neutrality ought not to be too far wrong.

The apparent absence of end-game effects in the data, justifying the convenient use of a stationary model, is not surprising given the nature of the stage games in this experiment. In the unequal position matches, which form the large majority of matches in the data in most rounds, the intuitive rule prescribes play of a one-shot Nash equilibrium. Thus, if an individual in such a match is convinced that his opponent will play according to the rule in the last round, there is no reason for the individual to deviate in the last round. In the penultimate round, if the individual does not expect a lastround position distribution that is much different than that of the steady state equilibrium and if his matched opponent occupies a different position than his own, similar considerations apply. For equal-position matches in the last round, theoretically there is the symmetric mixed equilibrium of the stage game, but we would hesitate to rely on that (or anything else) for a prediction. In fact, for the purpose of explaining the absence of end-game effects what is most important is only that whatever the subjects themselves predict about equal-position matched play in the last round does not influence incentives in the penultimate round when the subjects are matched with an opponent in an unequal position.

Our explanation for the monotonicity of compliance rates in $y$ in the data is related to the discussion of discounting above. We suspect that if the experiment could be controlled so that the parameters and discount factors were such that the intuitive rule did not form an equilibrium, our subjects would be at sea. Perhaps after a much longer play sequence they would manage to settle on one of the rules that do form equilibria, but this would not be something that their real-world experiences would help with. And in rounds 51-100 of such a treatment we have no idea what would happen. So, consider the thought experiment in which it is common knowledge that all players discount geometrically, but that each player's discount factor is a random 
draw from some distribution of discount factors, with positive probability on the event that the aggregate amount of discounting is insufficient for the intuitive rule to form an equilibrium (of the heterogeneous-population model with complete information about all discount factors). Our guess is that in such an experiment we would see some subjects attempting to play the rule and some subjects groping about; the lower the $y$ - value the less groping and the higher the compliance rate. In this sense then, the model (or at least some imagined variant of it) could explain the observed monotonicity in $y$.

Might there be alternative explanations for monotonicity of compliance rates in $y$ ? Perhaps. First, there is intuition for the idea that the higher is $y$ the more one is willing to risk the worst outcome for a chance at the best outcome. So, if an individual thinks that the matched opponent might play the intuitive rule with some error, the individual might be more likely to try to depart when $y$ is high than when it is low (although the necessary assumptions on the error-generating process might not be realistic). But it seems possible to us that this could be formalized anyway with a generalized version of the theoretical model of Section 3 and so is not necessarily an alternative to it. Second, if $y$ is high and an individual thinks the intuitive rule is being played, the individual may choose to sacrifice current payoff when in a lower position than that of the matched opponent by departing from the rule in order to get to a higher present-value position. But this is characteristic behavior of a high-discount factor player, independently of $y$. Such players will soon find themselves in the extreme state and will be in compliance with the intuitive rule after Round 50.

We noted earlier the pattern of departures from the intuitive rule in $\mathrm{BOS}(5)$ and $\mathrm{BOS}(10)$ in which a 4 meets a 4 , plays $\leftarrow$, then plays $\rightarrow$ against a 4 in the next round. In the simplistic terms of our discussion about discounting, it is as though the player first acted as though she had a discount factor no higher than the common one, then, in the next period had one that was higher. While we cannot rationalize this within the confines of our model, it does strike us as reminiscent of behavior in other experiments (see Ainslie and Haslam, 1992, for a survey) that is more consistent with a hypothesis of hyperbolic discounting, and hence time-inconsistency, than with geometric discounting.

Finally, we point out one of the other steady state equilibria of the model that has features that are reflected in some of the data. It is generated by a variant of the intuitive rule in which individuals act as though positions 3 and 4 are the same. In other words, when an individual in position 3 meets 
an individual in position 4, the first randomizes as though he were in position 4 and the second randomizes as though she were in position 3. It therefore has a chance to provide an explanation for the pattern of departures from compliance discussed in the previous paragraph. As an example, for BOS(5), such steady state equilibria exist for $\beta<.95$. For instance, when $\beta=.9$,

$$
\begin{gathered}
p_{00}=.78, p_{11}=.93, p_{22}=.83, p_{33}=p_{43}=.69, p_{44}=p_{34}=.64 . \\
\pi_{0}=.27, \pi_{1}=.09, \pi_{2}=.06, \pi_{3}=.12, \pi_{4}=.46 .
\end{gathered}
$$

This does well at explaining the position frequencies, but less well at explaining the lack of $\rightarrow$ play when pairs with equal current positions are matched. There are of course many other hybrids; however all of these would have to make substantial use of the intuitive rule to be consistent with the data. 


\section{A Appendix: Instructions to Subjects}

This is an experiment in decision-making. If you follow the instructions carefully and make good decisions, you can earn a considerable amount of money. You will be paid in private and in cash at the end of the session.

There are twelve people participating in this session. It is important that you remain silent once play has begun. If you do not, the experiment will be terminated, and you will receive none of your prize money. If you have a question during the experiment, raise your hand and a monitor will come over to where you are sitting and answer your question in private.

The experiment will consist of a simple game played 100 times in succession. In each of the 100 rounds you will be asked to click either the left arrow $(\rightarrow)$ or right arrow $(\leftarrow)$ on the screen with your mouse. Your winnings for the round will be $5 \phi, 20 \phi$, or $30 \phi$, depending on the choice you make and the choice made by your co-player for that round. Thus your total winnings for the experiment will be between $\$ 5.00$ and $\$ 30.00$. Since you will be paid in cash, privately, at the end of the experiment, none of the other participants in the experiment will know what your winnings are. You will also never be identified by name on any publications or reports on the results from this experiment.

In each round, your co-player will be one of the other experimental subjects in this room. They have all been recruited as subjects in the same way that you have, and they are reading exactly the same instructions that you are for the first time now. In each round your co-player will be chosen anew according to a completely random drawing. Thus, in each round your co-player is equally likely to be any of the other subjects, independently of anything that has transpired before that round. Neither you nor your co-player will be told the identity of the other.

Winnings for you and your co-player in each round are determined as follows: If both of you choose the right arrow $(\rightarrow)$, you both get $5 \phi$. If both of you choose the left arrow $(\leftarrow)$, you both get $5 \phi$. If one of you chooses $(\leftarrow)$ and the other chooses $(\rightarrow)$, the one who chose $(\rightarrow)$ gets $30 \notin$ and the other gets $20 \notin$. To summarize, your payoff table is

\begin{tabular}{|c|c|c|c|}
\cline { 2 - 3 } Own Choice & \multicolumn{3}{|c|}{ Co-Player's Choice } \\
\cline { 2 - 4 } & & $\leftarrow$ & $\rightarrow$ \\
\cline { 2 - 4 } & $\leftarrow$ & $5 \phi$ & $20 \phi$ \\
\cline { 2 - 4 } & $\rightarrow$ & $30 \phi$ & $5 \phi$ \\
\hline
\end{tabular}


All players have exactly the same payoff table.

Although the identities of matched co-players will not be revealed in any round, each of you will receive a piece of information about the other's recent play history before choosing which arrow to click. This piece of information is called the player's current position, which can be at any of the five locations on the line segment below.

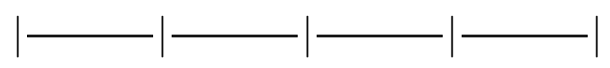

A player's current position is normally arrived at by taking his/her previous position and shifting it one location to the right if the player's previous choice was $(\rightarrow)$ or one location to the left if the previous choice was $(\leftarrow)$.

However, if the previous position was

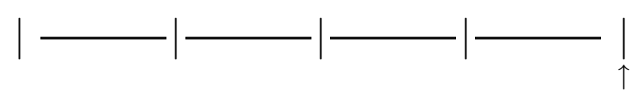

and the player chose $(\rightarrow)$, the new position will simply be the same as the previous position. Similarly, if the previous position was

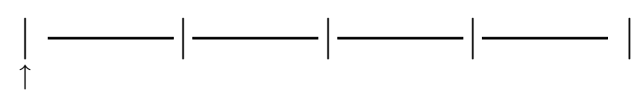

and $(\leftarrow)$ was chosen, the position does not change.

You will be reminded in each round as to your own current position as that is information that your co-player has about you. Each player will also be kept informed of the distribution of ALL players' positions. In round one, for instance, all 12 players will begin at the middle position, so the initial screen display will be: 
Round 1 of 100

Total Earnings so far $\$ 0$

OWN Current position

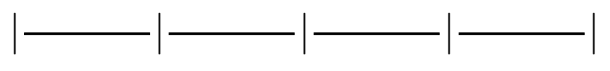

$\uparrow$

CO-PLAYER'S current position

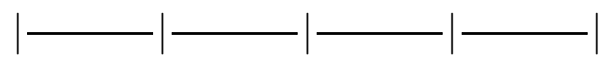

$\uparrow$

Current position frequencies

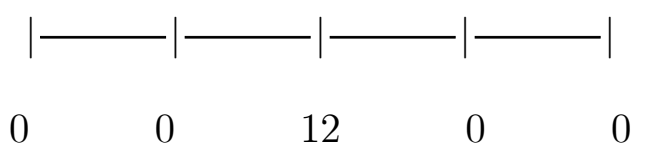

Payoff Table:

\begin{tabular}{|c|c|c|c|}
\hline \multirow{4}{*}{ Own Choice } & \multicolumn{3}{|c|}{ Co-Player's Choice } \\
\hline & & $\leftarrow$ & $\rightarrow$ \\
\hline & $\leftarrow$ & $5 \phi$ & $20 \phi$ \\
\hline & $\rightarrow$ & $30 \phi$ & $5 \phi$ \\
\hline
\end{tabular}

Please Choose an arrow

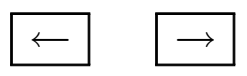

When all players in the room have made their choices in a round, your payoff for the round will be displayed in the form of a highlighted cell in the payoff table. To begin the next round, simply click the mouse anywhere on the screen.

Are there any questions? 


\section{References}

[1] Aumann, R. and S. Hart, "Long Cheap Talk", mimeo, 1999.

[2] Ainslie, G. and N. Haslam, "Hyperbolic Discounting", in Choice over Time, G. Loewenstein, ed., Russell Sage Foundation, New York, 1992.

[3] Barany, I., "Fair Distribution Protocols or How Players Replace Fortune", Mathematics of Operations Research, 17 (1992), 327-340.

[4] Ben-Porath, E. and E. Dekel, "Signaling Future Actions and the Potential for Sacrifice", Journal of Economic Theory, 57 (1992), 36-51.

[5] Brown, J. and R. Rosenthal, "Testing the Minimax Hypothesis: A Reexamination of O'Neill's Game Experiment", Econometrica, 58 (1990), 1007-1028.

[6] Cooper, R., R. Forsythe, D. DeJong, and T. Ross, "Alternative Institutions for Resolving Coordination Problems: Experimental Evidence on Forward Induction and Preplay Communication," in Coordination in Economic Activity, J. Friedman, ed., Kluwer Academic Press: Amsterdam, 1994.

[7] Kreps, D., and J. Sobel, "Signalling", Chapter 25 in Handbook of Game Theory, Volume 2, R. Aumann and S. Hart, eds., Elsevier, 1994.

[8] Meyer, D., J. van Huyck, R. Battalio, and T. Saving, "History's Role in Coordinating Decentralized Allocation Decisions: Laboratory Evidence on Repeated Binary Allocation Games", Journal of Political Economy, 100 (1992), 292-316.

[9] Mookherjee, D. and B. Sopher, "Learning Behavior in an Experimental Matching Pennies Game", Games and Economic Behavior, 7 (1994), $62-91$.

[10] Ochs, J., "The Coordination Problem in Decentralized Markets: an Experiment", Quarterly Journal of Economics, 105 (1990), 545-559.

[11] Rapoport, A., "Individual Strategies in a Market Entry Game", Group Decision and Negotiation, 4 (1995), 117-133. 
[12] Rapoport, A. and R. Boebel, "Mixed Strategies in Strictly Competitive Games: A Further Test of the Minimax Hypothesis", Games and Economic Behavior, 4 (1992), 261-83.

[13] Rapoport, A., D. Seale, and L. Ordonez, "Tacit Coordination in Large Groups with Both Strategic and Outcome Uncertainty", mimeo, 1999.

[14] Rosenthal, R. and H. Landau, "A Game-Theoretic Analysis of Bargaining with Reputations", Journal of Mathematical Psychology, 20 (1979), 233-255.

[15] Roth, A. and M. Malouf, "Game-Theoretic Models and the Role of Information in Bargaining", Psychological Review, 86 (1979), 574-594.

[16] Schelling, T., The Strategy of Conflict, Harvard university Press, Cambridge, 1960.

[17] Selten, R., A. Sadrieh, and K. Abbink, "Money Does Not Induce Risk Neutral Behavior but Binary lotteries Do Even Worse", Universitat Bonn Sonderforschungbereich 303, Discussion Paper B 343, 1995.

[18] Shachat, J., "Mixed Strategy Play and the Minimax Hypothesis", UCSD Economics Discussion Paper 96-37, 1996. 


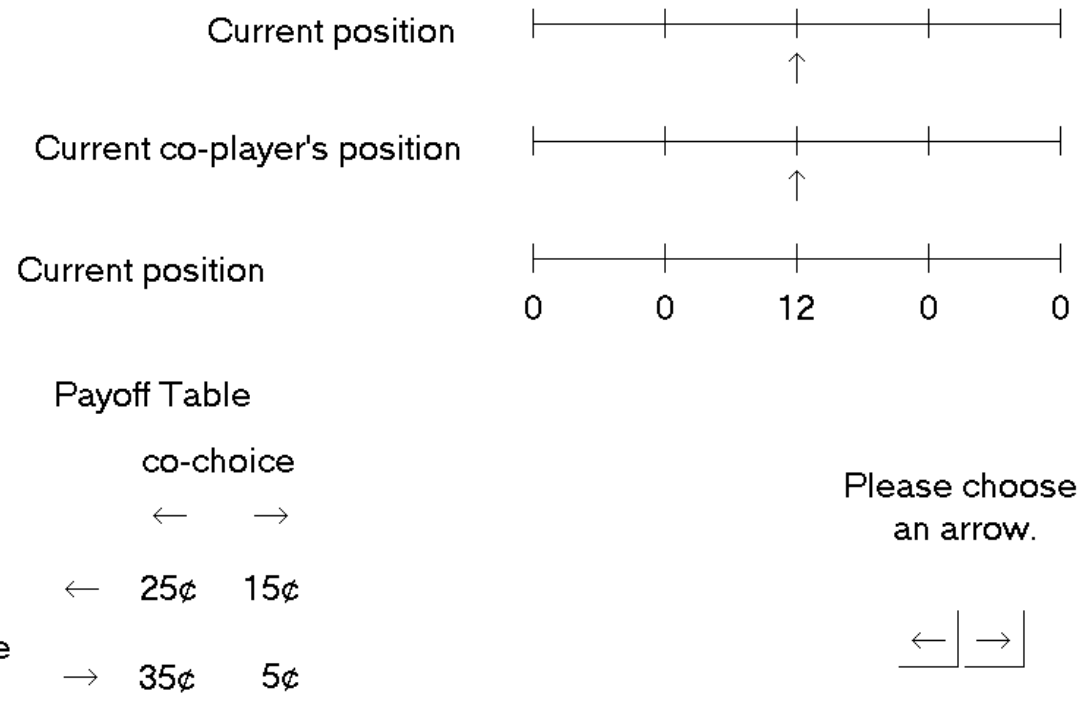

Figure 1: Sample Screen Image 


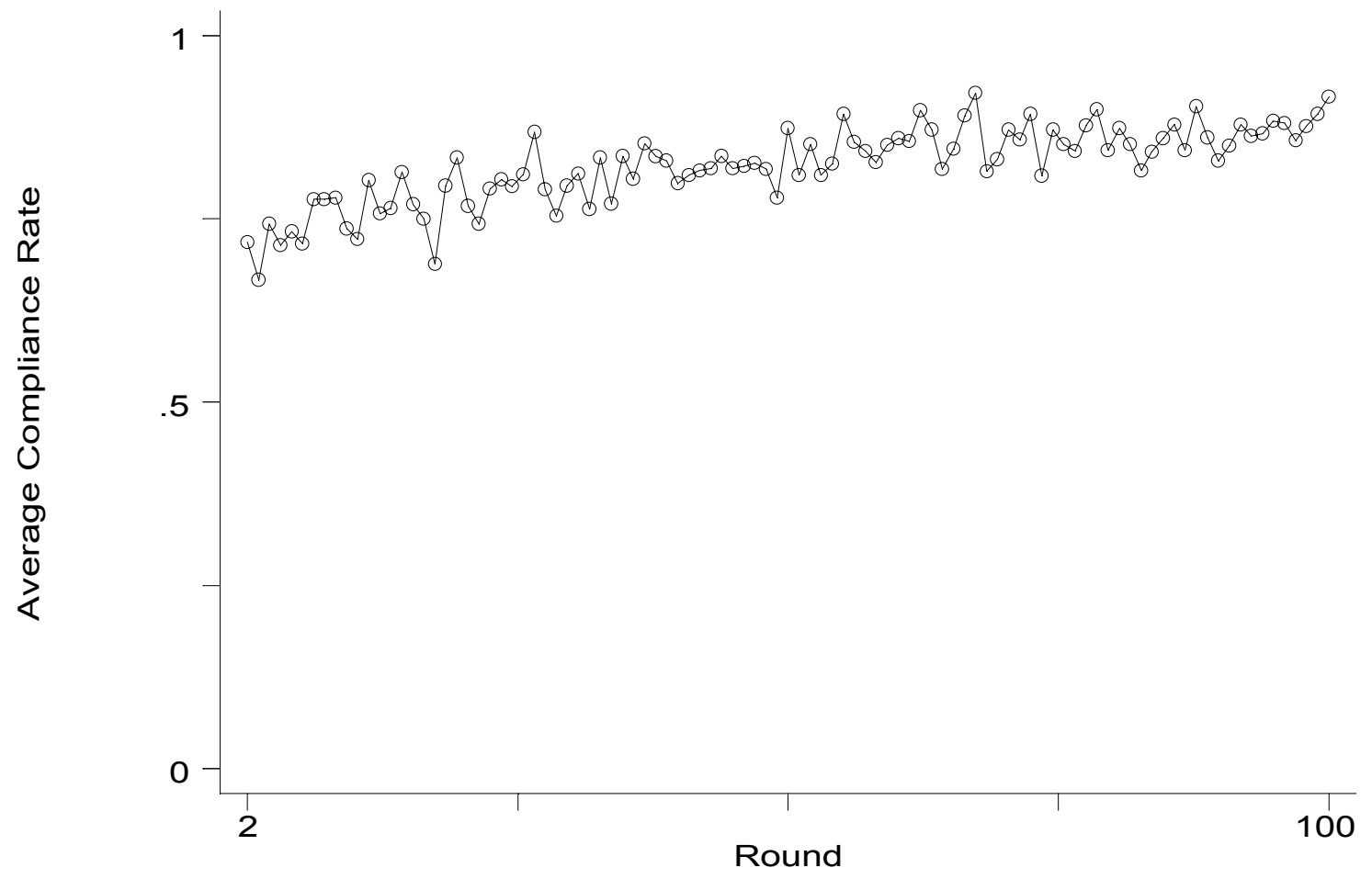

Figure 2: Average compliance rates by round -- all treatments 


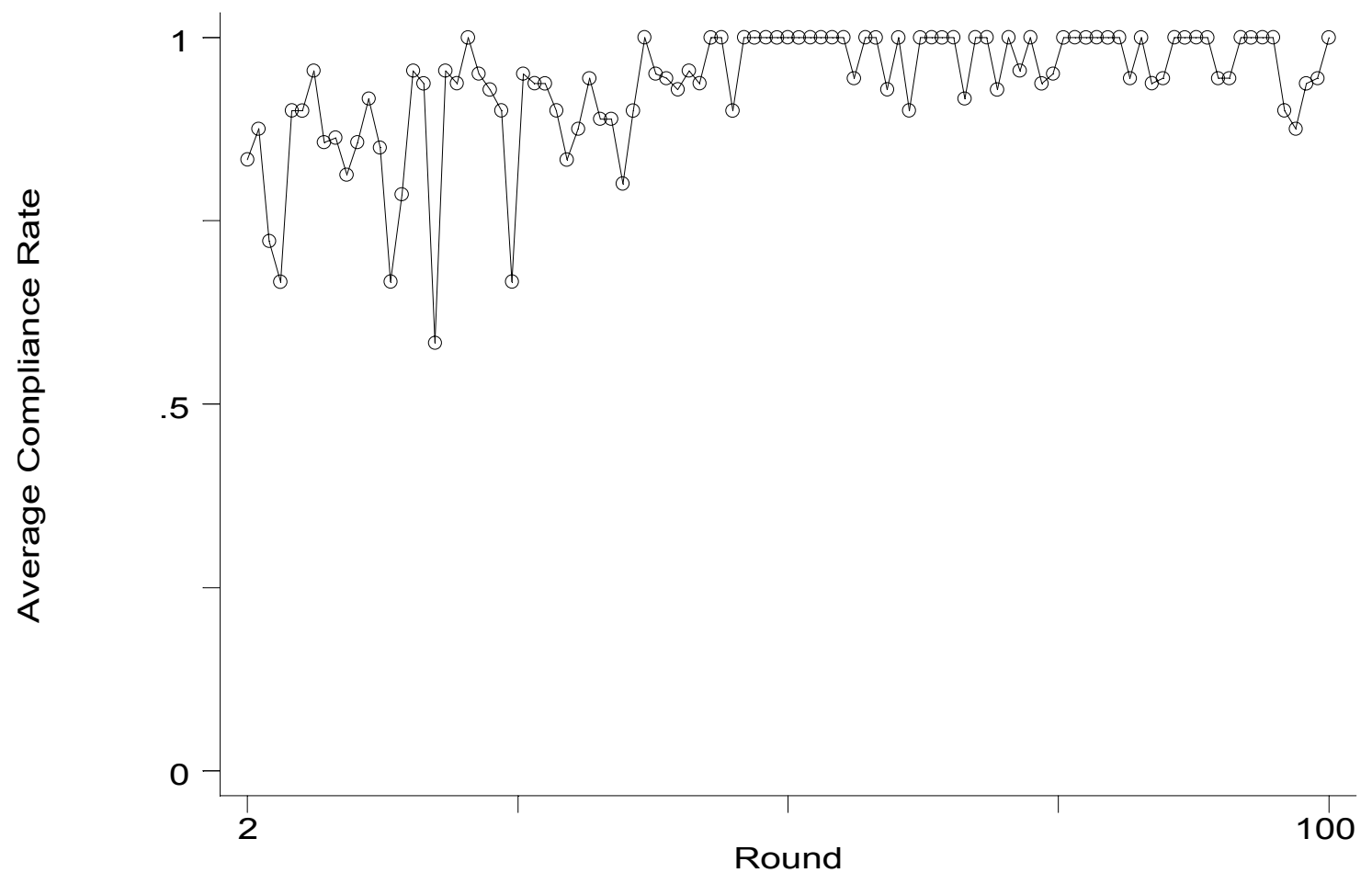

Figure 3: Average compliance rates by round - BOS(0)

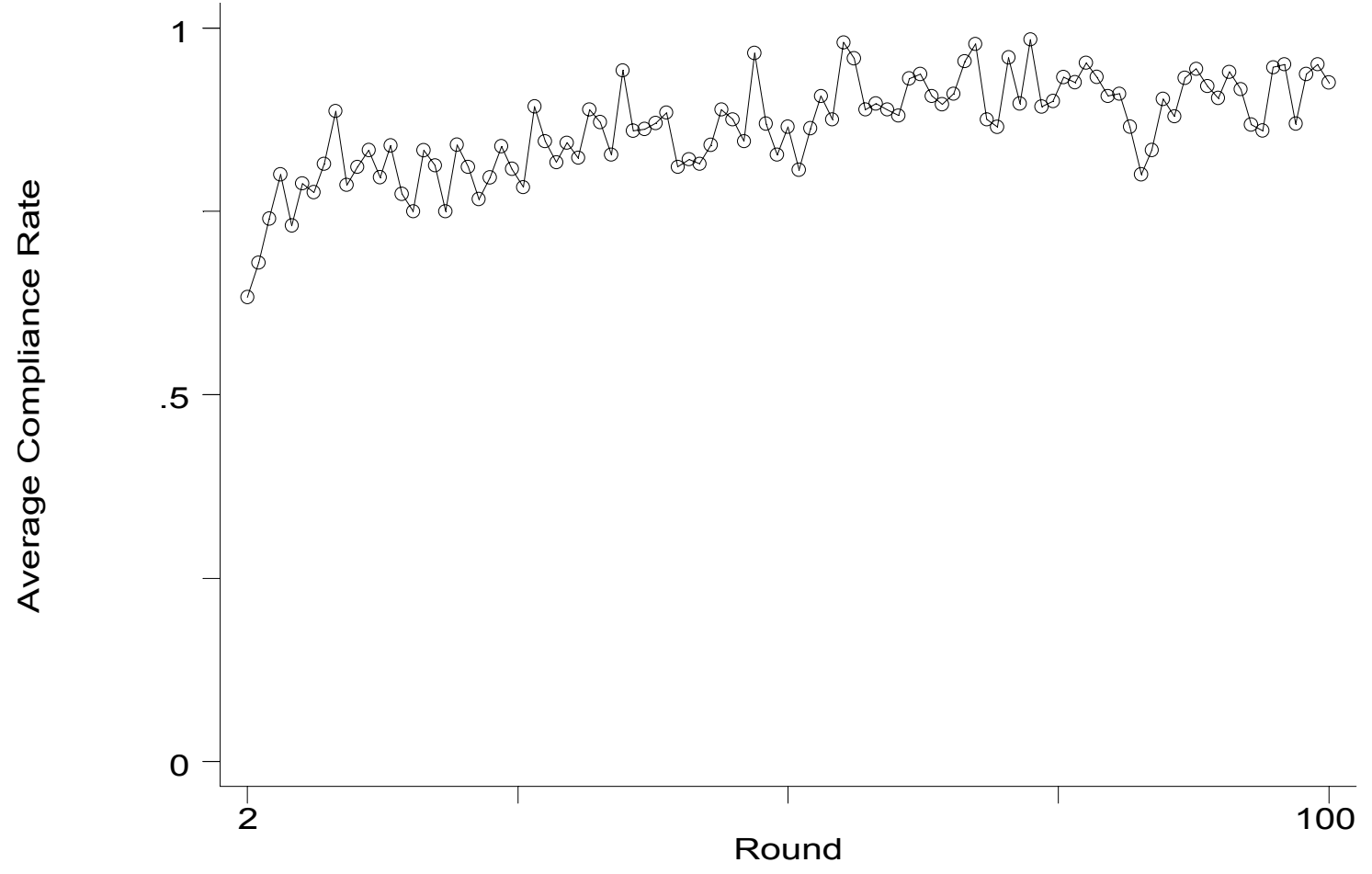

Figure 4: Average compliance rates by round - BOS(5) 


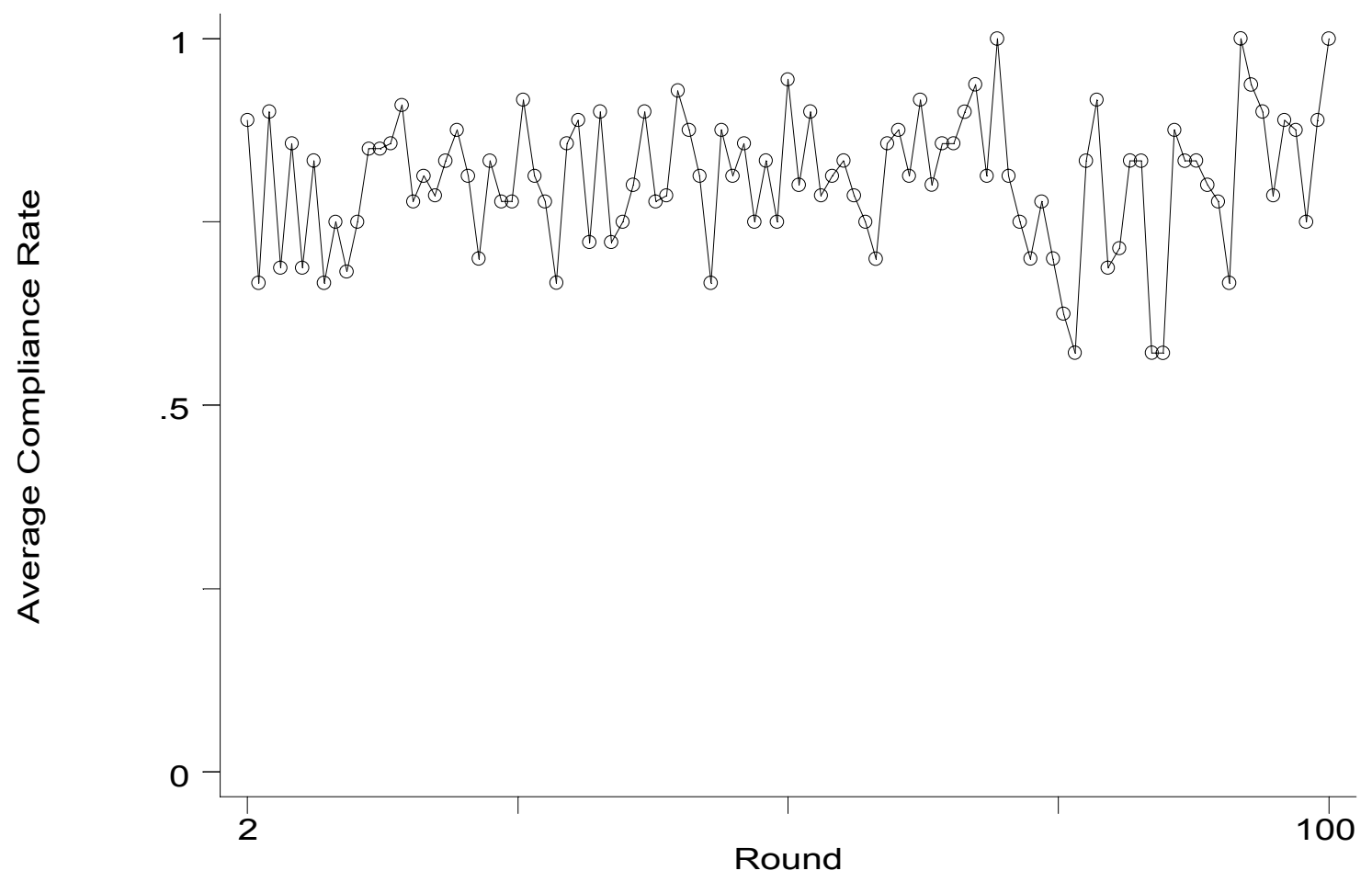

Figure 5: Average compliance rates by round - BOS(10)

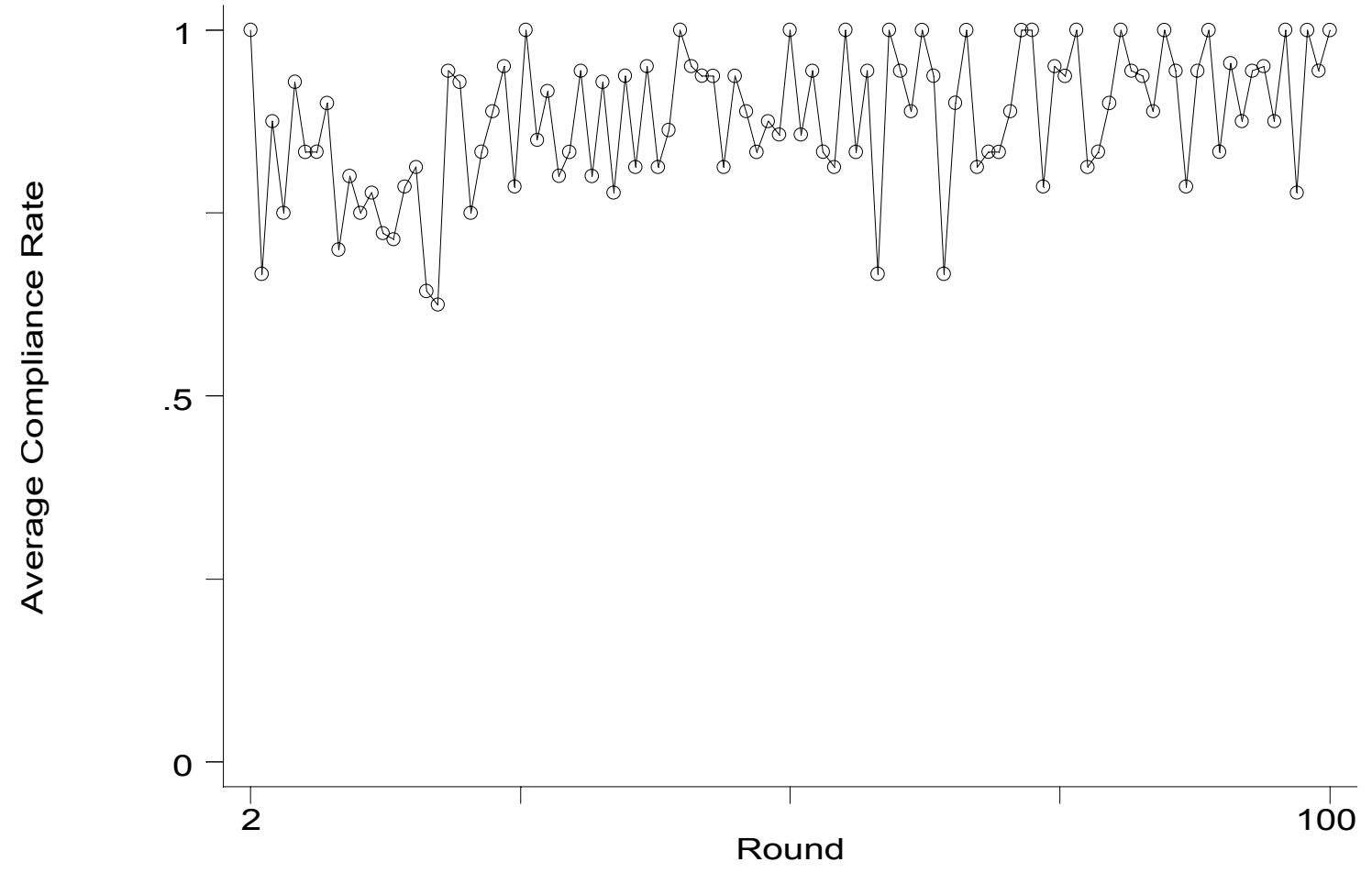

Figure 6: Average compliance rates by round - HD(5) 


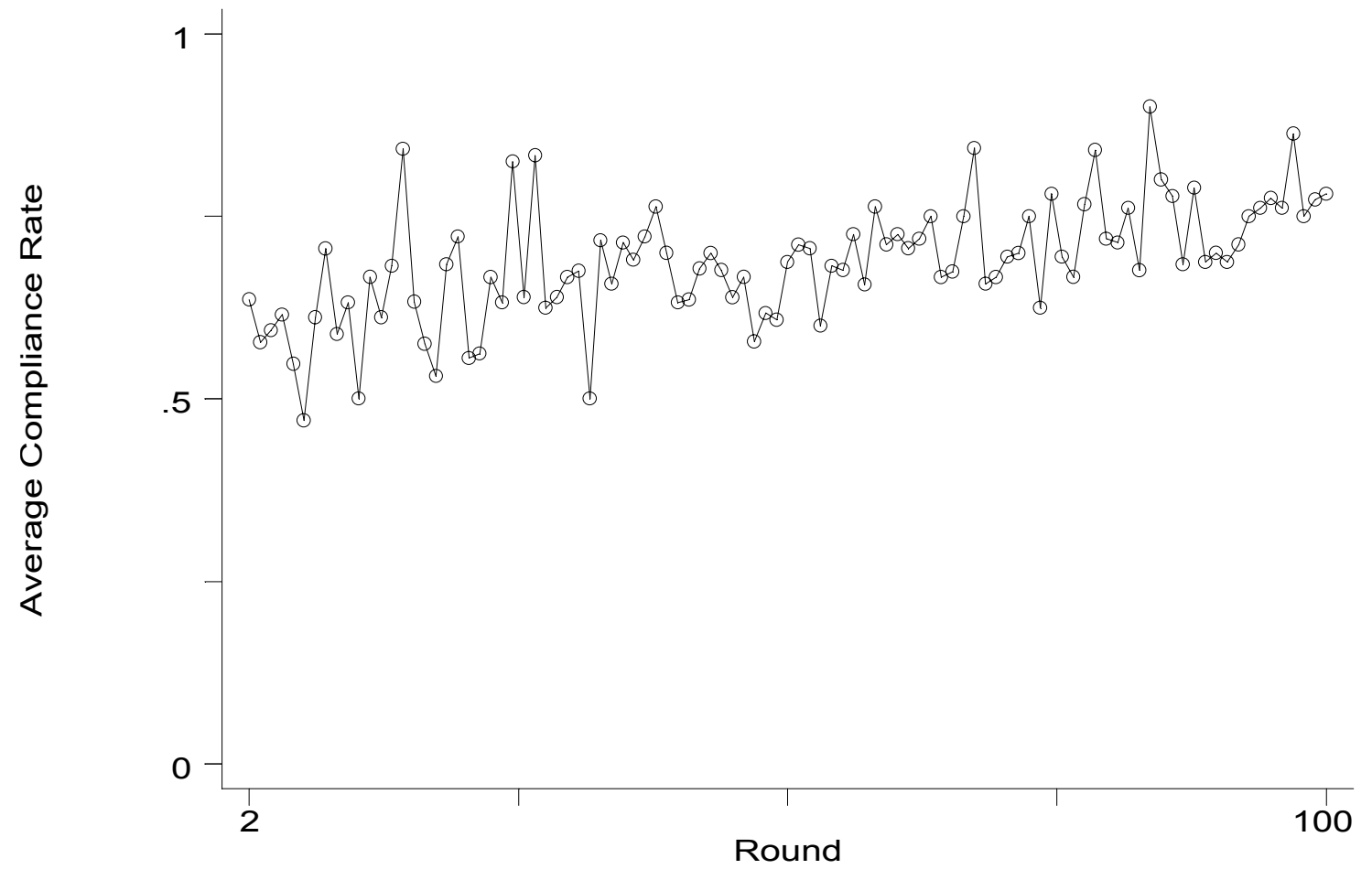

Figure 7: Average compliance rates by round - HD(10) 